COMMUNICATIONS IN

ANALYSIS AND GEOMETRY

Volume 14, Number 1, 59-89, 2006

\title{
Semistable Degeneration of Toric Varieties and Their Hypersurfaces
}

\begin{abstract}
SHENGDA Hu
We provide a construction of examples of semistable degeneration via toric geometry. The applications include a higher dimensional generalization of classical degeneration of $K 3$ surface into 4 rational components, an algebraic geometric version of decomposing $K 3$ as the fiber sum of two $E(1)$ 's as well as it's higher dimensional generalizations and many other new examples.
\end{abstract}

\section{Introduction.}

Surgery operation has been a central topic in every branch of topology and geometry. For example, in 3-dimension, geometrization conjecture states that one can classify 3-dimension into eight different "geometries" up to certain connected sum surgeries. For 4-manifold, we don't have such a classification conjecture precisely because we do not yet understand the surgeries of 4-manifolds. Unfortunately, connected sum is not a surgery in symplectic geometry. Instead, surgery theory in symplectic geometry is more subtle and influenced more by surgery in algebraic geometry. The latter is the main topic of this article.

There are primarily two types of surgeries in algebraic geometry. One class is birational transformation such as blow up-blow down, flip-flop. The birational geometry is a central topic in algebraic geometry. The famous Mori theory was designed to study this type of surgeries. Their importance in symplectic geometry was documented by many important papers [17], [15]. Another class of surgery comes from deforming the defining equation of algebraic varieties. Suppose that $F_{t}=\left\{f_{t}=0\right\}$ for $t \neq 0$ defines a family of smooth algebraic varieties. We can let $t \rightarrow 0$ such that $F_{0}=\left\{f_{0}=0\right\}$ acquires singularities. We further require the singularities of $F_{0}$ to be normal crossing. In this case, $F_{0}$ can be viewed as a union of smooth manifolds intersecting along (complex) codimension 1 submanifold. Namely, we "decompose" $F_{t}$ as a union of manifolds intersecting each other transversely. This is precisely what we mean surgery in topology. However, this is still 
too general to have any nice theory. Mumford proposed the notion of "semistable degeneration".

Definition 1.1. A degeneration is a morphism $\pi: X \rightarrow U \stackrel{\text { open }}{\subset} \mathbb{C}$ with $0 \in U$, such that the restriction $\pi: X \backslash \pi^{-1}(0) \rightarrow U \backslash\{0\}$ is smooth. $\pi$ is semistable, if $X$ is nonsingular and the fiber $\pi^{-1}(0)$ is reduced, with nonsingular components crossing normally (cf. [12] Ch II).

In many ways, semistable degeneration is the correct set-up to work with. For example, the monodromy is unipotent. Well-known Clemens-Schmidt exact sequence related cohomology of $F_{t}$ to that of $F_{0}$. Hodge structure has a nice limit at $F_{0}$. There is a theory of $\log$ differential forms on $F_{0}$. The list goes on.

In a different direction, semistable degeneration also appears in quantum cohomology. During last several years, a central topic in quantum cohomology is the surgery theory for Gromov-Witten invariants. In symplectic category, there are two different versions of surgery theory by Li-Ruan [15] and Ionel-Parker [11]. Recently, an algebro-geometric version analogous to that of Li-Ruan was obtained by J. Li [16]. These surgery theories have many important applications in quantum cohomology. The set-up in these surgery theories is precisely semistable degeneration. One of purposes of this article is to provide many more interesting examples to apply these surgery techniques, which the author hope to investigate in the future.

Although we have many beautiful theories for semistable degeneration, surprisingly, there are few concrete examples. Mumford proved a general theorem that every degeneration induces a semistable degeneration. However, Mumford's theorem is not constructive and not very useful in terms of constructing example. The only nontrivial example in author's knowledge is the semistable degeneration of $K 3$-surface into four copies of blow up of $\mathbb{C P}^{2}$ along 6-points. It is a beautiful construction and let us briefly describe it. We first take a degeneration of smooth degree 4 hypersurfaces of $\mathbb{C P}^{3}$ to a union of four hyperplanes, say a pencil. This pencil has base loci. We can blow up $\mathbb{C P}^{3}$ to resolve base loci. In this process, we increase the number of components in central fiber. Now the total space is a 3 -fold and Mori theory applies. We use Mori theory to blow down the total space. The resulting one is a semistable degeneration where the central fibers are four copies of the blow-up of $\mathbb{C P}^{2}$ along 6-points. In higher dimension, we do not have Mori theory. Hence, we do not know if there is a semistable degeneration of quintic Calabi-Yau 3-folds into five copies of blow-up of $\mathbb{C P}^{3}$. Our method in this article will provide such an example and much more. In fact, we 
will describe a general method to construct semistable degeneration of toric varieties and their hypersurfaces. Then, we will use our methods to construct many examples. Our examples include the classical example of $K 3$ and its natural generalizations into higher dimension such as Calabi-Yau hypersurface of $\mathbb{C P}^{n}$. Many other examples are also constructed.

Toric varieties can be thought of as compactifications of an algebraic torus $\left(\mathbb{C}^{\times}\right)^{n}$ and polytopes or fans are used to describe how the compactifications work. They contain many interesting varieties as subvariety. For example, most Calabi-Yau 3-folds known up to now are subvarieties of toric varieties. In principle, one could obtain everything about a toric variety from the fan or polytope that defines it. We consider here toric varieties that can be defined by a polytope (open or compact). In the case of compact polytope, it specifies not only the toric variety, but also a polarization, i.e. an embedding into some projective space. This will enable us to write down how the equations change when the toric variety (or its subvarieties) degenerates. Through polytopes, our construction connects back to symplectic geometry via the moment map - it can be thought of as "doing surgery with respect to the image of the moment map".

The first main result in this paper is that we can talk about semistable degeneration in toric category. Namely, the total space, the smooth fibers and the components in the central fiber are all toric varieties. The initial data is a subdivision $\Gamma$ of a nonsingular polytope $\triangle$ (thus $X$, the toric variety defined by $\triangle$, is nonsingular) and the theorem is

Theorem 3.1 If $\Gamma$ is a nonsingular semistable subdivision, then there exists a toric variety $\widetilde{X}$ and a map $p: \widetilde{X} \rightarrow \mathbb{C}$, such that $p$ is a semistable degeneration of $X$ to $p^{-1}(0)$. The dual graph of the fiber $p^{-1}(0)$ is given by the dual graph of the subdivision $\Gamma$ and a component in $p^{-1}(0)$ is the toric variety defined by the corresponding subpolytope in $\Gamma$.

Given a semistable degeneration $p: \widetilde{X} \rightarrow \mathbb{C}$, we can consider a smooth subvariety $\widetilde{V}$ in $\widetilde{X}$. Suppose that $\widetilde{V}$ intersects transversely with general fiber of $p$ as well as all the components in the fiber $p^{-1}(0)$. Then in a small neighbourhood of 0 , the restriction of $p$ to $\widetilde{V}$ gives a semistable degeneration. In our case, we also see that the components in $\left(\left.p\right|_{\widetilde{V}}\right)^{-1}(0)$ are subvarieties of toric varieties. One application is

Theorem 4.7 There is a semistable degeneration of degree $n+1$ Calabi-Yau hypersurface in $\mathbb{C P}^{n}$, such that all the components in the singular fiber are rational and the dual graph of the central fiber is the triangulation of $S^{n-1}$ by the boundary of n-simplex. 
Another result also concerning Calabi-Yau hypersurface is a generalization of realizing $K 3$ surface as fiber-sum of two $E(1)$ 's.

Theorem 4.8 There exists a semistable degeneration of Calabi-Yau hypersurface in $\mathbb{C P}^{2 k+1}$ into two components $Y_{i}(i=1,2)$, such that $Y_{1} \cong Y_{2} \cong Y$ satisfy the following: $\left(K_{Y}\right)^{2}=0,-K_{Y}$ is effective and defines a Calabi-Yau subvariety $Z$ of $Y, Z$ is isomorphic to a hypersurface in $\mathbb{C P}^{k} \times \mathbb{C P}^{k}$ and the singular loci of the central fiber is $Z$.

The idea of studying families using subdivision of polytope has appeared in the literature, e.g. [1]. Alexeev has completely different purpose of constructing the completion of an arbitrary one-parameter family of stable toric pair and semi-stability was not an issue. In particular, semi-stability was not an issue in [1]. For compact polytope, our construction fits well with the GKZ construction [8] and Chow quotients and families [13] [14]. In particular, it gives geometric description for some facets of secondary polytopes.

The paper is organized as following: $\S 2$ describes the construction of

the polytope $\widetilde{\triangle}$. The main theorem 3.1 is proven in $\S 3$. The examples are in $\S 4$. $\S 5$ describes a generalization of the construction to give semistable degenerations with higher dimensional base.

\section{Acknowledgments}

The paper is based on my Ph.D. thesis and I want to thank my advisor, Professor Yongbin Ruan, for his constant guidance and encouragement. I'd like to thank Mark Gross for pointing out to author the paper [1] by $\mathrm{V}$. Alexeev and $\mathrm{Yi} \mathrm{Hu}$ for pointing out the relation to Chow quotient and Chow families. I benefitted a lot from discussions with Professor Miles Reid, Rajesh Kulkarni, Wojciech Wieczorek, Mainak Poddar and Bohui Chen. I want to thank them all here.

\section{Semistable subdivision of polytopes.}

This is a purely combinatoric section. The main result is theorem 2.8 and we won't need the details of the proofs in the rest of the paper.

Let $M$ and $N$ be dual lattices of rank $n$ and $\langle\cdot, \cdot\rangle$ denote the pairing between them. We consider a (not necessarily compact) simple lattice polytope $\triangle$ in $M_{\mathbb{R}}$ :

$$
\triangle=\left\{v \in M_{\mathbb{R}} \mid\left\langle v, n_{i}\right\rangle \geq-a_{i}, n_{i} \in N, a_{i} \in \mathbb{Z} \text { for } i=1, \ldots, s\right\},
$$

with all vertices in $M$. Let $\Gamma$ be a (polyhedral) subdivision of $\triangle$ into union of subpolytopes $\left\{\triangle_{j}\right\} . \Gamma$ is called simple if all $\triangle_{j}$ 's are simple polytopes. An 
open $l$-face $\sigma$ of $\triangle_{j}$ will also be called $l$-face of $\Gamma$ and be denoted by $\sigma \prec \Gamma$. We make an exception for the 0 -faces of $\triangle$ by declaring they are not a 0 -face of $\Gamma$. The restriction of $\Gamma$ to $\sigma \prec \triangle$ is defined to be the subdivision formed by $\left\{\triangle_{j} \cap \sigma\right\}$ and denoted by $\Gamma \cap \sigma$, thus the faces of $\Gamma \cap \sigma$ are also faces of $\Gamma$. When not explicitly stated, all faces are relative open faces while polytopes are closed polytopes.

Definition 2.1. A simple subdivision $\Gamma$ is semistable if for any $l$-face $\sigma$ of $\Gamma$ and $k$-face $\tau$ of $\triangle$, if $\sigma \subset \tau$, then there are exactly $k-l+1 \triangle_{j}$ 's such that $\sigma \prec \triangle_{j}$.

Example 2.2. Let $M=\mathbb{Z}$ and $\triangle$ be the line segment between 0 and $n \in \mathbb{Z}$. Any subdivision with subdivision points in $\mathbb{Z}$ is semistable.

Example 2.3. Let $M=\mathbb{Z}^{2}$ and $\triangle$ be the convex hull of the points $(0,0)$, $(3,0)$ and $(0,3)$. For the subdivisions shown in figure 1 , the first two are semistable while the last one is not.

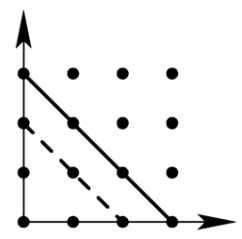

(a)

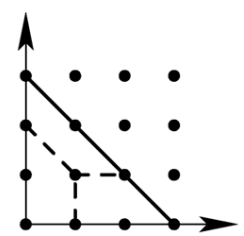

(b)

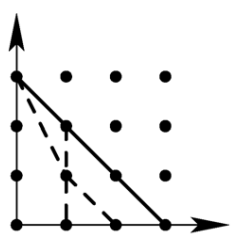

(c)

Figure 1.

Proposition 2.4. If $\cap_{i=1}^{l} \triangle_{j_{i}} \cap \sigma_{k} \neq \emptyset$, for a $k$-face $\sigma_{k} \prec \triangle$, then it has dimension $k-l+1$.

Proof: We prove it in three steps.

Step 1: If $\cap_{k=1}^{l} \triangle_{j_{k}} \neq \emptyset$, then it has dimension $n-l+1$.

First suppose $l=2$. Assume the opposite, i.e., $\triangle_{i} \cap \triangle_{j} \neq \emptyset$ has dimension $s<n-1$. Let $\tau$ be an $s$-face of $\Gamma$ that is contained in the intersection. Suppose $\tau$ lies in a $t$-face $\sigma_{t}$ of $\triangle$, then $\tau$ is a face of $t-l+1 \triangle_{k}$ 's. Since $\triangle$ is simple polytope, $\sigma_{t}$ is a face of $n-t(n-1)$-faces of $\triangle$. On the other hand, there are $n-s(n-1)$-faces of $\triangle_{i}$ having $\tau$ as a face and at most $n-t$ of them could lie on $(n-1)$-faces of $\triangle$, which left $t-s$ of them contained in the unique $n$-face of $\triangle$. From assumption, $\triangle_{i}$ and $\triangle_{j}$ have no common $(n-1)$-faces. Then $\tau$ has to be a face of $t-s \triangle_{k}$ 's other than $\triangle_{i}$ or $\triangle_{j}$. In all, $\tau$ is a face of $t-l+2 \triangle_{k}$ 's, contradiction. 
For general $l$, the $l-1$ intersections $\triangle_{j_{1}} \cap \triangle_{j_{k}}(k=2, \ldots, l)$ are $(n-1)$ faces of $\triangle_{j_{1}}$, which has non-empty intersection. They have exactly one common $(n-l+1)$-face by property of simple polytope.

Step 2: If $\triangle_{i} \cap \sigma_{k} \neq \emptyset$ for a $k$-face $\sigma_{k} \prec \triangle$ then it has dimension $k$.

Suppose $\triangle_{i} \cap \sigma_{k} \neq \emptyset$. Let $\tau \prec \Gamma$ be a $s$-face contained in the intersection. Then $\tau$ is a face of exactly $k-s+1 \triangle_{j}$ 's, say, $\left\{\triangle_{i}, \triangle_{j_{1}}, \ldots \triangle_{j_{k-s}}\right\}$. By proposition 2.4, in the $n-s+1(n-1)$-faces of $\triangle_{i}$ that have $\tau$ as a face, $k-s$ of them are the intersections with $\triangle_{j_{t}}$ 's, which left $n-k$ of those lie on the $n-k(n-1)$-faces of $\Delta$ that $\sigma_{k}$ is a face of. So there is a $k$-face of $\triangle_{i}$ having $\tau$ as a face contained in $\sigma_{k}$. In particular, the subdivision $\Gamma \cap \sigma_{k}$ is semistable.

Step 3: Apply step 1 to the semistable subdivision $\Gamma \cap \sigma_{k}$.

Proposition 2.5. $\Gamma$ is semistable then for any vertex $p$ of $\Gamma$ which is not a vertex of $\triangle$, there are exactly $n+1$ edges $\sigma_{0} \ldots, \sigma_{n}$ of $\Gamma$ such that $p \prec \sigma_{i}$.

Proof: First assume that $p$ is in the interior of $\triangle$, then $p$ is vertex of $n+1$ subpolytopes, say $\triangle_{j=0 \ldots, n}$. For each of the edges of $\Gamma$ that $p$ is a vertex of, there are $n$ of the $\triangle_{j}$ 's intersecting along it. The proposition follows.

Suppose $p \subset \sigma$ for $\sigma$ a $k$-face of $\triangle$, then $p$ is vertex of $k+1$ subpolytopes, say $\triangle_{j=0, \ldots, k}$. Since $\Gamma \cap \sigma$ is semistable, $p$ is vertex of $k+1$ edges of $\Gamma \cap \sigma$. By proposition 2.4, the $\triangle_{j}$ 's intersect along the closure of a $(n-k)$-face of $\Gamma$, which gives $n-k$ edges of $\Gamma$ that have $p$ as a vertex. Adding the numbers we finishes the proof.

We define the dual simplicial complex $K_{\Gamma}$ associated to a semistable simple subdivision $\Gamma$ as following. The set of vertices is the set of polytopes in subdivision $\Gamma$. For each $l$-face $\sigma$ in $\Gamma$ that lies in the interior of $\triangle$, an $(n-l)$-simplex $\kappa$ is defined by the polytopes that $\sigma$ is a face of. It's easy to see that $K_{\Gamma}$ is contractible since $\triangle$ is contractible.

Let $\Gamma$ be a semistable subdivision and $p \prec \Gamma$ be a vertex contained in an $l$-face $\tau$ of $\triangle$. Then there are $l+1$ edges $\sigma_{0}, \ldots, \sigma_{l}$ of $\Gamma \cap \tau$ such that $p \prec \sigma_{i}$ $(i=0, \ldots, l)$. For $i=i, \ldots, l$, let $\sigma_{i}(p)$ denote the primary vector of $\sigma_{i}$ at $p$, then they satisfy a single integral relation $w_{0} \sigma_{0}(p)+\ldots+w_{l} \sigma_{l}(p)=0$ for some $w_{i}>0$. Reorder the $w_{i}$ 's such that $w_{1} \leq w_{2} \leq \ldots \leq w_{l}$ and call the primary vector $W_{p}=\left(w_{0}, \ldots, w_{l}\right) \in \mathbb{Z}^{l+1}$ the weight vector of $\Gamma$ at $p$. Recall that a vertex $p \prec \triangle$ is nonsingular if the primary vectors of the edges meeting $p$ form a basis of the lattice $M$.

Definition 2.6. A vertex $p \prec \Gamma$ is balanced if $W_{p}=(1, \ldots, 1)$, is nonsingular if $p$ is nonsingular in all sub-polytopes containing $p$. A semistable 
subdivision is balanced (resp. nonsingular) if all it's vetices are balanced (resp. nonsingular). Suppose $K_{\Gamma}$ is l-dimensional, maximal vertices of $\Gamma$ are the vertices of $\Gamma$ that lie in some $l$-face of $\triangle$. A balanced $\Gamma$ is mildly singular if all its maximal vertices are nonsingular.

Obviously, $p$ is balanced if $p \prec \Gamma$ is contained in an edge of $\triangle$. Also clear from the definition, any intersection among the subpolytopes of $\Gamma$ contains at least one maximal vertex. The last sentence of the definition makes sense due to the following:

Proposition 2.7. For semistable subdivision $\Gamma$, nonsingular vertex $p \prec \Gamma$ is balanced.

Proof: Let $\tau$ be the $l$-face of $\triangle$ that contains $p$. First suppose $l=n$, i.e. $\tau$ is the interior of $\triangle$. Let $\sigma_{0}, \ldots, \sigma_{n}$ be the $n+1$ edges of $\Gamma \cap \tau$ such that $p \prec \sigma_{i}$ $(i=0, \ldots, n)$. Since $\rho$ is nonsingular, we can write $\sigma_{0}(p)=\sum_{i=1}^{n} \pm \sigma_{i}(p)$. Now the signs are all negative since $p$ is an interior point of $\triangle$.

For general $l$, the result follows from applying the above to $\Gamma \cap \tau$.

Theorem 2.8. Let $\Gamma$ be mildly singular. There exist concave piecewise linear continuous functions $F$ on $\Gamma$. Let $\widetilde{\triangle}=\{(y, x) \mid y \geq F(x)\} \subset \mathbb{R} \times \triangle \subset$ $\mathbb{R} \times M_{\mathbb{R}} . F$ is unique upto addition of affine functions when we require $F$ be integral and

$\widetilde{\triangle}$ be nonsingular over the maximal vertices of $\Gamma$.

If furthermore $\triangle$ and $\Gamma$ are nonsingular, then $\widetilde{\triangle}$ is nonsingular.

Proof: Suppose $\sigma=\triangle_{i} \cap \triangle_{j}$ be a $(n-1)$-face of $\Gamma$. Let $p \prec \sigma$ be a maximal vertex, then there are $n+1$ edges $\tau_{0}, \ldots, \tau_{n}$ of $\Gamma$ that meet at $p$. Suppose that $\tau_{0} \nprec \triangle_{i}$, while $\tau_{1} \nprec \triangle_{j}$. Let $f_{i j, p}$ be the rational affine function which defines the hyperplane that contains $\sigma$ and $f_{i j, p}\left(p+\tau_{0}(p)\right)=1$. By definition, $f_{i j, p}=-f_{j i, p}$. For another maximal vertex $q \prec \sigma$, we can define $f_{i j, q}$ similarly. Since $p$ and $q$ are both nonsingular we have $f_{i j, p}=f_{i j, q}=: f_{i j}$.

Let $\mathcal{A}=\left\{f: M_{\mathbb{R}} \rightarrow \mathbb{R} \mid f\right.$ affine and $\left.f(M) \subset \mathbb{Q}\right\}$ be the group of all rational affine functions on $M_{\mathbb{R}}$. $\mathcal{A}$ can be identified with $\mathbb{Q}^{n+1}$. Let $C\left(K_{\Gamma}\right)$ be the oriented chain complex of $K_{\Gamma}$ and define $\alpha \in \operatorname{Hom}\left(C_{1}\left(K_{\Gamma}\right), \mathcal{A}\right)$ by $\alpha\left(\kappa_{\triangle_{i} \cap \triangle_{j}}\right)=f_{i j}$ on the basis and extend linearly to the whole $C_{1}\left(K_{\Gamma}\right)$. It follows from definition of $f_{i j}$ that $\alpha$ is a cocycle.

Because $K_{\Gamma}$ is contractible, there exists $\beta \in \operatorname{Hom}\left(C_{0}\left(K_{\Gamma}\right), \mathcal{A}\right)$, such that $\delta(\beta)=\alpha$. Specifically, $\beta$ assigns a function $f_{i} \in \mathcal{A}$ to each sub-polytope $\triangle_{i}$ 
in $\Gamma$, such that $f_{j}-f_{i}=f_{i j}$ if $\triangle_{i} \cap \triangle_{j} \neq \emptyset$. Suppose $\delta \beta^{\prime}=\alpha$ as well, let $\gamma=\beta-\beta^{\prime}$ then $\delta \gamma=0$, i.e. $\gamma$ assigns the same function $f \in \mathcal{A}$ to all the sub-polytopes in $\Gamma$. We define $F: \triangle \rightarrow \mathbb{R}$ by $\left.F\right|_{\triangle_{i}}=f_{i}$. Then $F$ is a piecewise linear continuous function on $\Gamma$. For concavity, we only need to recognize that $F$ can also be defined as $F(x)=\max _{i} f_{i}(x)$. Obviously, the $F$ we just constructed satisfies (2.2).

Uniqueness is now easy to see. Let $F^{\prime}$ be another function satisfying (2.2), then the cochain $\beta^{\prime} \in \operatorname{Hom}\left(C_{0}\left(K_{\Gamma}, \mathcal{A}\right)\right)$ defined by $F^{\prime}$ satisfies $\delta \beta^{\prime}=\alpha$ as well.

The rest of the theorem follows straightforwardly from the construction. For example, $\widetilde{\triangle}$ is simple by proposition 2.5 and the fact that there is a vertical edge over each vertex of $\triangle$.

Remark 2.9. The function $F$ is called a lifting function of $\Gamma$ and the polytope $\widetilde{\triangle}$ constructed in the theorem is an open lifting of $\triangle$ by $\Gamma$. Note that $\triangle$ may be non-compact.

Remark 2.10. The following is obvious. If $\Gamma \cap \sigma$ is mildly singular for some closed face $\sigma \prec \triangle$, the function $\left.F\right|_{\sigma}$ and $\tilde{\triangle} \cap(\sigma \times \mathbb{R})$ satisfies the conclusion of the theorem.

Remark 2.11. Sometimes it's more convenient to work with compact polytopes instead of open ones. In that case, we can consider $\widetilde{\triangle}^{c}=$ $\widetilde{\triangle} \cap\{(y, x) \mid y \leq\langle a, x\rangle+b\}$ for $a \in N$ and sufficiently big $b \in \mathbb{Z}$. We say that $\triangle^{c}$ is a compact lifting of $\triangle$ by $\Gamma$.

Remark 2.12. Suppose $\triangle$ is compact and $\operatorname{dim} K_{\Gamma}>1$. Let $(\triangle, \triangle \cap M)$ be a pair as in [8] then the theorem implies that mildly singular subdivision $\left\{\left(\triangle_{i}, \triangle_{i} \cap M\right)\right\}$ is coarse, i.e. corresponds to a facet of the secondary polytope of $\triangle$.

\section{Semistable degeneration of toric varieties.}

We prove the main result (theorem 3.1) and give some examples. There is also a similar result (theorem 3.5) on the degeneration of toric varieties with orbifold singularities. As a result of the construction, we give concrete description of the degenerations in remark 3.3.

We first recall some toric geometry and fix the notations. Standard references for toric geometry are [5], [7] and [18]. Let $M, N$ and $\triangle$ be as in $\S 2$ and $\Sigma$ be the normal fan of $\triangle$ in $N_{\mathbb{R}}$. For a (rational strongly convex 
polyhedra) cone $\alpha$ in $N_{\mathbb{R}}=N \otimes_{\mathbb{Z}} \mathbb{R}$, its dual cone in $M_{\mathbb{R}}$ is denoted by $\alpha^{\vee}$. The toric variety $X=X_{\Sigma}=X_{\triangle}$ is defined by gluing together the affine varieties $X_{\sigma}=\operatorname{Spec}\left(\mathbb{C}\left[\sigma^{\vee} \cap M\right]\right)$, where $\sigma$ is a cone of $\Sigma$. Let $\Sigma^{\prime}$ be a fan $\Sigma^{\prime}$ in $N_{\mathbb{R}}^{\prime}$. A toric morhpism $\phi_{*}: X_{\Sigma^{\prime}} \rightarrow X_{\Sigma}$ is given by homomorphism of lattices $\phi: N^{\prime} \rightarrow N$ which induces map on fans, i.e. for each cone $\sigma^{\prime}$ in $\Sigma^{\prime}$ there is some cone $\sigma$ in $\Sigma$ such that $\phi_{\mathbb{R}}\left(\sigma^{\prime}\right) \subset \sigma$. Let $|\Sigma|$ be the support of $\Sigma$, i.e. the union of the cones in $\Sigma$, then

$$
\phi_{*} \text { is proper } \Longleftrightarrow \phi^{-1}(|\Sigma|)=\left|\Sigma^{\prime}\right| \text {. }
$$

Recall that the dual graph of the central fiber of a semistable degeneration is defined by assigning a vertex for each component and a $k$-simplex whenever $k$ components having non-empty intersection.

Theorem 3.1. Suppose $\triangle$ and subdivision $\Gamma$ are nonsingular, then there exists a semistable degeneraiton $p: \widetilde{X} \rightarrow \mathbb{C}$ of $X$ to $p^{-1}(0)$. The dual graph $G$ of the fiber $p^{-1}(0)$ is isomorphic to $K_{\Gamma}$ and a component in $p^{-1}(0)$ is the toric variety defined by the corresponding subpolytope in $\Gamma$.

Proof: By theorem 2.8, let $\widetilde{\triangle}$ be a nonsingular open lifting of $\triangle$ by $\Gamma$. Let $\widetilde{\Sigma}$ be the fan of $\widetilde{\triangle}$, then $\Sigma_{\Gamma}$ is a subfan of $\widetilde{\Sigma}$. Let $\widetilde{X}$ be the toric variety defined by $\widetilde{\Sigma}$, then $\widetilde{X}$ is smooth. Note that by construction, $|\widetilde{\Sigma}| \subset \overline{\mathbf{H}}=N_{\mathbb{R}} \times \overline{\mathbb{R}^{+}}$. The cones $\alpha_{\sigma}$ that correspond to the vertical faces $\sigma$ of $\widetilde{\triangle}$ lie in $N_{\mathbb{R}} \times\{0\}$ and all the other cones in $\widetilde{\Sigma}$ lie in $N_{\mathbb{R}} \times \mathbb{R}^{+}$. Let $\Lambda$ be the fan in $\mathbb{R}$ with $|\Lambda|=\overline{\mathbb{R}^{+}}$, then the variety it defines is $\mathbb{C}$.

Consider the exact sequence of lattices:

$$
0 \rightarrow N \stackrel{i}{\rightarrow} N \oplus \mathbb{Z} \stackrel{\mu}{\rightarrow} \mathbb{Z} \rightarrow 0,
$$

where $\mu$ is the projection to the second summand and $i$ is the inclusion. The maps are compatible with the fans $\Sigma, \widetilde{\Sigma}$ and $\Lambda$ and define a sequence of proper morphisms:

$$
X \stackrel{i_{*}}{\rightarrow} \widetilde{X} \stackrel{p}{\rightarrow} \mathbb{C},
$$

from which it follows that the fiber of $p$ over the generic point of $\mathbb{C}$ is isomorphic to $X$. The rays of $\widetilde{\Sigma}$ in $\mathbf{H}$ is mapped to the open positive cone of $\mathbb{R}$, thus $p$ maps the divisors defined by such rays to 0 , i.e. $p^{-1}(0)=\cup_{i=1}^{k} X_{i}$, where $X_{i}$ 's are the divisors defined by such rays. By construction, $X_{i}$ 's are themselves toric varieties which is defined by the subpolytopes in the subdivision $\Gamma$. The statement about the dual graph of the fiber $p^{-1}(0)$ is obvious. 
Next we write down the map $p$ in the affine pieces. Dualizing (3.1), we have exact sequence:

$$
0 \rightarrow \mathbb{Z} \stackrel{j}{\rightarrow} M \oplus \mathbb{Z} \stackrel{\nu}{\rightarrow} M \rightarrow 0
$$

where $j$ is the inclusion and $\nu$ is the projection to the first summand. Suppose $\widetilde{\triangle}$ is defined by the lifting function $F$. Let $q \prec \Gamma$ be a vertex which is an inner point of $\triangle$ and $\widetilde{q}$ be the lift of it in $\widetilde{\triangle}$. Let $\sigma_{0}, \ldots, \sigma_{n}$ be the $n+1$ edges of $\widetilde{\triangle}$ that meet at $\widetilde{q}$ and $\alpha_{q}$ be the cone dual to $\widetilde{q}$, then $\alpha_{q}^{\vee}$ is generated by $\sigma_{0}(\widetilde{q}), \ldots, \sigma_{n}(\widetilde{q})$. Let $X_{q}$ be the affine piece defined by $\alpha_{q}^{\vee}$. By the definition of $F$ in theorem 2.8 , we have $\sum_{k=0}^{n} \sigma_{k}(\widetilde{q})=(0, \ldots, 0,1)=j(1)$. Thus the map $j$ induces $j_{*}: \mathbb{C}[t] \rightarrow \mathbb{C}\left[x_{0}, \ldots, x_{n}\right], t \mapsto \prod_{k=0}^{n} x_{k}$, where $x_{k}$ 's are the coordinates given by the edges of $\alpha_{q}^{\vee}$. It follows that the map $p$ on the affine piece $X_{q}$ is defined by $\left(x_{0}, \ldots, x_{n}\right) \mapsto \prod_{k=0}^{n} x_{k}$.

Now suppose $q \prec \Gamma$ is a vetex which is an inner point of a $0<r$ dimensional face $\tau \prec \triangle$. It follows from above and remark 2.10 that $j_{*}$ is defined by $t \mapsto \prod_{k=0}^{r} x_{k}$ and $p$ is defined by $\left(x_{0}, \ldots, x_{n}\right) \mapsto \prod_{k=0}^{r} x_{k}$ in the affine piece $X_{q}$.

If $q$ is a vertex of $\triangle$, then it is a face of a vertical edge $\sigma_{0}$ of $\widetilde{\triangle}$. Thus $j_{*}(t)=x_{0}$ and $p\left(x_{0}, \ldots, x_{n}\right)=x_{0}$ in the affine piece $X_{q}$.

Since the affine pieces as described above cover the central fiber, the theorem follows.

The theorem doesn't require $X$ (or $\triangle$ ) to be compact. In the following remarks we'll make the assumption that $X$ (and $\triangle$ ) be compact.

Remark 3.2. If we take a compact lifting, then instead of (3.2), we get sequence of morphisms of compact toric varieties:

$$
X \stackrel{i_{*}^{c}}{\longrightarrow} \widetilde{X}^{c} \stackrel{p^{c}}{\longrightarrow} \mathbb{C P}^{1}
$$

Remark 3.3. When $\triangle$ is compact, we are in fact constructing a degeneration of polarized toric varieties. The degeneration can be put in more concrete terms using the embedding of $X$ into $\mathbb{C P}^{\ell-1}$ given by the polariation (recall that $\ell=|\triangle \cap M|$ ). Suppose $F$ and $F^{\prime}$ are lifting functions that give rise to nonsingular liftings of $\triangle$ as in theorem 2.8, then $F^{\prime}=F+G$ for some integral affine function $G$. Let $\widetilde{i}_{F}:\left(\mathbb{C}^{\times}\right)^{n+1} \rightarrow \mathbb{C} \mathbb{P}^{\ell-1} \times \mathbb{C}$ be the embedding defined by

$$
\widetilde{x}=(x, \lambda) \mapsto\left(\left[\lambda^{F\left(\mathbf{m}_{1}\right)} x^{\mathbf{m}_{1}}: \ldots: \lambda^{F\left(\mathbf{m}_{\ell}\right)} x^{\mathbf{m}_{\ell}}\right], \lambda\right)
$$


Let $\widetilde{i}_{F^{\prime}}$ be the similar embedding defined by $F^{\prime}$. Then $\widetilde{i}_{F}$ and $\widetilde{i}_{F^{\prime}}$ has the same images since $\lambda \neq 0$. Let $\widetilde{X}_{F}$ be the closure of the image of $\widetilde{i}_{F}$. It follows that $\widetilde{X}=\widetilde{X}_{F}$ is independent of the lifting and it's the same $\widetilde{X}$ as in theorem 3.1. The map $p$ is given by

$$
p:\left(\left[\lambda^{F\left(\mathbf{m}_{1}\right)} x^{\mathbf{m}_{1}}: \ldots: \lambda^{F\left(\mathbf{m}_{\ell}\right)} x^{\mathbf{m}_{\ell}}\right], \lambda\right) \mapsto \lambda .
$$

Suppose $\Gamma$ subdivision $\triangle$ into $\cup_{i=0}^{k} \triangle_{i}$ and let $X_{i}$ be the toric variety defined by $\triangle_{i}$. By adding an affine function, we may assume that $\left.F\right|_{\triangle_{0}}=0$, then $F(\mathbf{m})>0$ for $\mathbf{m} \notin \triangle_{0}$ by concavity. Let $\left[y_{1}: \ldots: y_{\ell}\right]$ be the homogeneous coordinates of $\mathbb{C P}{ }^{\ell-1}$ and define

$$
H_{i}=\left\{\left[y_{1}: \ldots: y_{\ell}\right] \mid y_{j}=0 \text { if } \mathbf{m}_{j} \notin \triangle_{i}\right\} \quad \text { for } i=0, \ldots, k .
$$

Let $\ell_{i}=\left|\triangle_{i} \cap M\right|$, then $H_{i} \cong \mathbb{C P}^{\ell_{i}-1}$. Taking limit of the embedding $\widetilde{i}_{F}$ as $\lambda \rightarrow 0$, we get the embedding of $X_{0}$ into $\mathbb{C P}^{\ell_{0}-1} \cong H_{0}$. Using different presentations of $\widetilde{X}$ by using different lifting functions and taking limit as $\lambda \rightarrow 0$, we see that $p^{-1}(0)=\cup_{i=0}^{k} X_{i}$.

Remark 3.4. Recall that the Chow polytope of polarized toric variety $X_{\triangle}$ is identical to the secondary polytope of $\triangle$ (cf. [14]). We may interpret our construction as an explicit presentation of the Chow family over an affine piece of the Chow quotient of $\mathbb{C P}^{\ell-1}$. The description in remark 3.3 can also be seen as a special case of "perturbing, translating and specializing" in [10].

In case of $\triangle$ being singular, we can still formulate a similar result using the concept of "weak semistable degeneration" as in [2]. The difference between weak semistable and semistable degenerations is that the total space in the first case need not to be nonsingular. The degeneration we construct from a singular simple toric variety could not have nonsingular total space, since $\triangle$ is not nonsingular, while we can still require the subdivision to be mildly singular.

Theorem 3.5. Suppose $\Gamma$ is a mildly singular semistable subdivision of simple $\triangle$, then there exists a weak semistable degeneration $p: \widetilde{X} \rightarrow \mathbb{C}$ of $X$ to $p^{-1}(0)$. The dual graph $G$ of the fiber $p^{-1}(0)$ is isomorphic to $K_{\Gamma}$ and a component in $p^{-1}(0)$ is the toric variety defined by the corresponding subpolytope in $\Gamma$.

Proof: The proof goes word by word as the proof of theorem 3.1, except for the fact that $\widetilde{X}$ might be singular. The reducibility of the central fiber follows from the fact that $\Gamma$ is balanced. 
Example 3.6. The semistable degeneration for the subdivision in example 2.2 is the result of successively blowup smooth points in the central fiber of $\mathbb{C} \mathbb{P}^{1} \times \mathbb{C}$.

Example 3.7. In example 2.3, (a) corresponds to blowup one point in the central fiber of $\mathbb{C P}^{2} \times \mathbb{C}$. The proper transformation of the central fiber is then $\widetilde{\mathbb{C P}^{2}} \cong \mathbb{P}(\mathcal{O}(-1) \oplus \mathbf{1})$. Blowing-up a fiber in $\mathbb{P}(\mathcal{O}(-1) \oplus \mathbf{1})$, we get the degeneration corresponding to $(b)$.

Example 3.8. The construction as in example 3.7 can be generalized to $\mathbb{C P}^{n}$. Let $e_{0}=(-1,-1, \ldots,-1) \in \mathbb{Z}^{n} \subset \mathbb{R}^{n}$ and $\left\{e_{i}\right\}$ be the standard integral basis of $\mathbb{Z}^{n}$. Let $\triangle(n)$ be the polytope in $\mathbb{R}^{n}$ spanned by $e_{0}$ and $e_{0}+(n+1) e_{i}, i=1, \ldots, n$, then $\triangle(n)$ is the standard reflexive polytope that represents $\mathbb{C P}^{n}$. Let $v_{0}=e_{1}, v_{i}=e_{i+1}-e_{i}$ for $i=1, \ldots, n$, where $e_{n+1}=0$. Let $\Sigma(n)$ be the maximal fan generated by $\left\{v_{i}\right\}_{i=0}^{n}$, then $\Sigma(n)$ is complete and nonsingular. $\triangle(n) \cap \Sigma(n)$ gives a nonsingular semistable subdivision $\Gamma(n)$ of $\triangle(n)$. There are $(n+1)$ subpolytopes in $\Gamma(n)$ and let $\triangle_{i}(n)$ denote the subpolytope containing $e_{i}$. The equations of the hyperplanes that bound $\triangle_{0}(n)$ are:

$$
\sum_{i=1}^{j} x_{i}=0, \text { for } j=1, \ldots, n \text {. }
$$

Figure 2 shows the subdivision $\Gamma(3)$ for $\mathbb{C P}^{3}$.

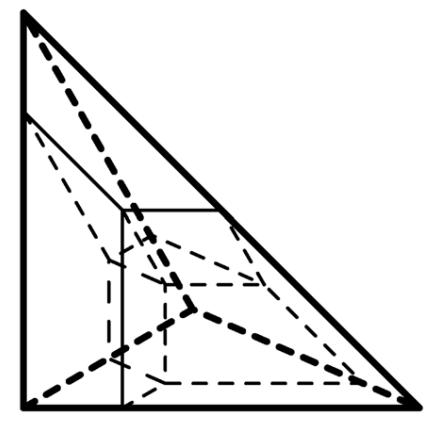

Figure 2.

The semistable degeneration constructed from $\Gamma(n)$ will then have $n+1$ components in the central fiber. The degeneration can also be viewed as the result of blowing-up smooth subvarieties in the central fiber of the trivial 
family as following. Let $\mathcal{F}$ be a complete flag in $\mathbb{C P}^{n}$, i.e. a chain of linear subspaces $p t=\mathcal{F}_{0} \subset \mathcal{F}_{1} \subset \ldots \subset \mathcal{F}_{n-1}$ where $\mathcal{F}_{i}$ has dimension $i$. Blow-up $\mathcal{F}_{i}$ in the central fiber starting from $i=0$ and then blow-up the proper transformation of $\mathcal{F}_{i+1}$, until all the $\mathcal{F}_{i}$ 's are blown-up. By symmetry of the subdivision, we see that all the components in the resulting central fiber are isomorphic to $Q^{n}$, the proper transformation of $\mathbb{C P}^{n}$ after all the blow-ups. Figure 3 is an illustration of the polytopes for $Q^{i}, i=1,2,3$.

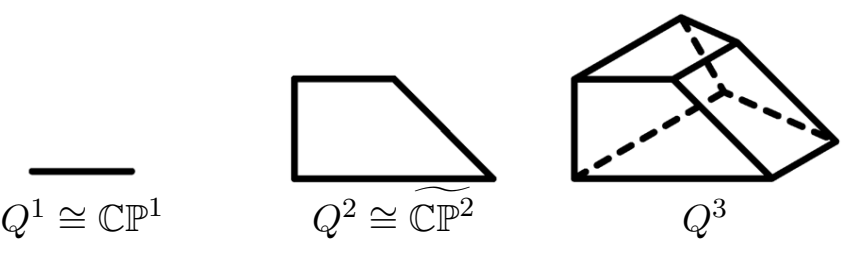

Figure 3.

Example 3.9. We can similarly define a weak semistable degeneration of weighted projective space. Let $\triangle$ be the polytope in $\mathbb{R}^{4}$ spanned by $e_{0}$, $e_{0}+8 e_{1}$ and $e_{0}+4 e_{i}$ for $i=2,3,4$, then $\triangle$ is the reflexive polytope representing the weighted projective space $\mathbb{P}(1,1,2,2,2)$. We use the fan $\Sigma(4)$ as described above to give a semistable subdivision of $\triangle$. The resulting subdivision is no longer symmetric, since $\triangle$ is not symmetric to begin with. It's not hard to show that the subdivision is balanced. It's mildly singular because the only maximal vertex $(0,0,0,0)$ is nonsingular.

Example 3.10. It is not true that all the semistable degeneration constructed from the theorem can be constructed also by blowing up the central fiber of a trivial family. The subdivision in figure 4 is a semistable subdivision, while the degeneration is not composition of blowing-ups on the central fiber.

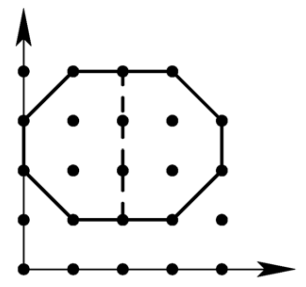

Figure 4 . 
Example 3.11. For an example of non-compact variety let $\triangle=M_{\mathbb{R}}$ or equivalently

$$
\triangle=\left\{m \in M_{\mathbb{R}} \mid\langle m, 0\rangle \geq 0\right\} .
$$

Thus the fan of $\triangle$ is $\Sigma=\{0\}$, the trivial fan in $N_{\mathbb{R}}$. The corresponding toric variety is simply the torus $\left(\mathbb{C}^{\times}\right)^{n}$. Let $\Gamma$ be the subdivision defined by the fan $\Sigma(n)$ as in example 3.8, then it's nonsingular. The semistable degeneration defined by $\Gamma$ is the familiar map $p: \mathbb{C}^{n+1} \rightarrow \mathbb{C}$, where $p\left(x_{0}, x_{1}, \ldots, x_{n}\right)=$ $\prod_{i=0}^{n} x_{i}$.

\section{Semistable degeneration of toric hypersurfaces.}

Let $p: X \rightarrow \mathbb{C}$ be a semistable degeneration and $V \subset X$ be a subvariety of $X$. Suppose $V$ intersects transversely with the central fiber. Let $p_{V}=\left.p\right|_{V}$ : $V \rightarrow \mathbb{C}$ and $V_{\lambda}=p_{V}^{-1}(\lambda)$. Then over a small neighbourhood $U \subset \mathbb{C}$ of 0 , $p_{V}$ is a semistable degeneration of $V_{\lambda}$. The construction in the last section provides a nice way to get semistable degeneration of toric hypersurfaces and complete intersections.

This section is organized as following. In $\S 4.1$ we describe two basic constructions to obtain semistable degenerations of toric subvarieties from the toric degeneration. The construction with open polytopes is more explicit, while the construction with compact polytopes is more general. $\S 4.2$ proves a lemma needed later to determine the components in the singular fiber of the degenerations. The examples start with $\S 4.3$, in which we describe the semistable degeneration of degree $d$ surfaces in $\mathbb{C P}^{3}$ into a chain of rational components. We obtain in $§ 4.3 .3$ an algebraic geometric version of the fiber-sum operation of two copies of $E(1)$ to get $K 3$ surface and in $\S 4.4 .2$ its generalization to higher dimension (theorem 4.8). In $\S 4.4 .1$, we recover the classic semistable degeneration of $K 3$ surfaces into four rational components and generalize it to degenerations of higher dimensional Calabi-Yau hypersurfaces in projective spaces (theorem 4.7). The section ends with $\S 4.4 .3$, in which we briefly describe a weak semistable degeneration of Calabi-Yau hypersurface of $\mathbb{P}(1,1,2,2,2)$.

\subsection{Basic constructions.}

4.1.1. Construction with open polytopes. Let $\triangle \subset \mathbb{R}^{n}$ be a nonsingular polytope and $X$ be the toric variety defined by $\triangle . \triangle$ also defines a very ample divisor $D_{\triangle}$ in $X$. Let $\Gamma$ be a nonsingular semistable subdivision

of $\triangle$. We first consider a generic hypersurface $V$ in the linear system $\left|D_{\triangle}\right|$. 
Referring to remark 3.3, $V$ is given by the intersection of the image of $i_{\triangle}$ with a generic hyperplane in $\mathbb{C P}^{\ell-1}$, where $\ell=\left|\Delta \cap \mathbb{Z}^{n}\right|$ is the number of lattice points in $\triangle$. We can thus write down the equations of $V_{\lambda} \subset X$ over a nonzero $\lambda$. Fix a generic hyperplane in $\mathbb{C P}^{\ell-1}$ :

$$
H=\left\{\left[y_{1}: \ldots: y_{\ell}\right] \mid \sum_{j=1}^{\ell} a_{j} y_{j}=0\right\} .
$$

The intersection of the image of $\widetilde{i}$ with $H$ as subvarieties in $\mathbb{C P}^{\ell-1} \times \mathbb{C}$ gives the total space $\widetilde{V}$ of the degeneration. Thus the equation of $V_{\lambda}$ is given by:

$$
\phi_{\lambda}=\sum_{j=1}^{\ell} \lambda^{F\left(\mathbf{m}_{j}\right)} a_{j} x^{\mathbf{m}_{j}}=0 .
$$

We may again assume that $\left.F\right|_{\triangle_{0}}=0$ for some subpolytope $\triangle_{0}$. Let $X_{0}$ (and $D_{0}$ ) be the toric variety (resp. the divisor on $X_{0}$ ) defined by $\triangle_{0}$. Taking limit when $\lambda \rightarrow 0$, we see that the central fiber $V_{0}$ contains as a component a generic hypersurface of $X_{0}$ in the linear system $\left|D_{0}\right|$.

4.1.2. Construction with compact polytopes. We may consider more general hypersurfaces and complete intersections by looking at compact lifting $\widetilde{X}^{c}$ of $\triangle$ by $\Gamma$. Then by $(3.4), p^{c}$ gives a family over $\mathbb{C P}^{1}$. We'll drop the superscript ${ }^{c}$ in this paragraph. Suppose $\widetilde{D}$ is a divisor on $\widetilde{X}$, such that $|\widetilde{D}|$ is generated by sections. Then, a generic hypersurface in $|\widetilde{D}|$ is smooth and it intersects transversely with generic fiber of $p$. Over a neighbourhood of $0 \in \mathbb{C P}^{1}$, we have a semistable degeneration of $V_{\lambda}^{\prime}=V^{\prime} \cap p^{-1}(\lambda)$. We use $\rho$ to denote the vertical faces in $\widetilde{\triangle}, \sigma$ to denote the faces from the subdivision $\Gamma$ and $\tau$ to denote the face at infinity and we'll abuse the notation to denote the faces in $\triangle$ as $\rho$ as well. Then $D_{\tau}$ is the class of a generic fiber. Suppose $\widetilde{D}=\sum_{\rho} a_{\rho} D_{\rho}+\sum_{\sigma} a_{\sigma} D_{\sigma}+a_{\tau} D_{\tau}$ and let $D=\sum_{\rho} a_{\rho} D_{\rho}$, then $V_{\lambda}^{\prime}$ is in the linear system $|D|$ of $X$. There is an obvious relation in $A_{n}(\widetilde{X})$ :

$$
D_{\tau}=\sum_{\sigma} D_{\sigma}
$$

Suppose $\left\{\sum_{\rho} \alpha_{\rho}(i) D_{\rho}=0 \mid i=1, \ldots, n\right\}$ are the relations in $A_{n-1}(X)$. Then, besides the obvious one, all the relations in $A_{n}(\widetilde{X})$ are given in

$$
\left\{\sum_{\rho} \alpha_{\rho}(i) D_{\rho}=\sum_{\sigma} \beta_{\sigma}(i) D_{\sigma} \mid i=1, \ldots, n\right\} \text { for some } \beta_{\sigma}(i) .
$$

Using these relations, we can get information on the components $C_{i}$ in the central fiber $V_{0}^{\prime}$. 


\subsection{A Lemma.}

Before the examples, we need a lemma to identify the components in the central fibers. Suppose $X$ is a smooth projective variety of dimension $n$. Let $L$ be a Cartier divisor on $X$ and $\mathcal{L}$ be the corresponding line bundle over $X$, with a generic section $s$. Let $\pi: E=\mathbb{P}_{X}(\mathcal{L} \oplus \mathbf{1}) \rightarrow X$ be the projectivization of $\mathcal{L}$ and $f$ be the class of the fiber. Let $\lambda=\left[\lambda_{0}: \lambda_{1}\right] \in \mathbb{C P}^{1}$ then $\left(\lambda_{1} s, \lambda_{0}\right)$ are sections of $\mathcal{L} \oplus \mathbf{1}$. Let $V_{0}$ (resp. $V_{\infty}, V_{\lambda}$ ) be (the closure of) the image of $(0,1)$ (resp. $\left.(s, 0),\left(\lambda_{1} s, \lambda_{0}\right)\right)$ in $E$. $V_{\lambda}$ are sections of the $\mathbb{C P}^{1}$ bundle $E$. As divisors in $E$ :

$$
V_{\lambda \neq[1: 0]}=V_{\infty}+\pi^{-1}(L), \quad \text { and }\left.\quad V_{0}\right|_{V_{0}}=L .
$$

Let $D$ be an ample divisor in $X$ such that $L+D$ is also ample and $\mathcal{D}$ be the corresponding line bundle. Let $s_{D}\left(\right.$ resp. $\left.s_{D}^{\prime}\right)$ be a generic section of $\mathcal{D}$ (resp. $\mathcal{L} \otimes \mathcal{D}$ ) and $\mathcal{L}_{D}=(\mathcal{L} \oplus \mathbf{1}) \otimes \mathcal{D}=\mathcal{L} \otimes \mathcal{D} \oplus \mathcal{D}$, then $s_{D}$ (resp. $s_{D}^{\prime}$ ) defines a generic hypersurface $D_{\infty}\left(\right.$ resp. $\left.D_{0}\right)$ in the linear system $|D|($ resp. $|L+D|)$. Let $T=D_{0} \cap D_{\infty}$, then $T$ is smooth codimension 2 subvariety of $X$ since everything is generic. Consider the sections $s_{\lambda, D}=\left(\lambda_{1} s_{D}^{\prime}, \lambda_{0} s_{D}\right)$ of $\mathcal{L}_{D}$. Let $W_{\lambda}$ be the closure of the image of $s_{\lambda, D}$ in $E \simeq \mathbb{P}_{X}\left(\mathcal{L}_{D}\right)$, then for $\lambda \neq 0, \infty$,

$$
W_{\lambda} \cap V_{0}=D_{0}, \quad W_{\lambda} \cap V_{\infty}=D_{\infty}, \text { and } W_{\lambda} \cdot f=1
$$

Apply Theorem IV-23 and Proposition IV-25 in [6], we see that $\left.\pi\right|_{W_{\lambda}}: W_{\lambda} \rightarrow$ $X$ is the blow-up of $X$ along $T$ for $\lambda \neq 0, \infty$. Denote the blow-up as $\tilde{X}_{T}$.

We claim that (4.1) essentially characterizes the blow-up of $X$ along $T$ as divisor in $E$.

Lemma 4.1. Suppose $R$ is a divisor in $E$ that satisfies (4.1), where the first two equations will be replaced by the corresponding intersections of divisor classes. Then a generic hypersurface $W$ in the linear system $|R|$ is the blow-up of $X$ along $T$, where $T$ now is interpreted as intersection of generic hypersurfaces of $X$ in the linear systems $|D|$ and $|D+L|$.

Proof: By (4.1), $W$ is generically a section of $E$ over $X$ and $W$ has at least 2 points over the points in $T$. Thus $W$ contains the whole fibers over points in $T$. The restriction of $\pi$ to $W$ is surjective and $\pi^{-1}(T)=\left.E\right|_{T}$ is Cartier in $W$. By the universal property of blow-up, $\left.\pi\right|_{W}$ factors through a unique morphism $\phi: W \rightarrow \widetilde{X}_{T}$. Well, $\phi$ has to be isomorphism since $W$ and $\widetilde{X}_{T}$ have identical exceptional loci. 
For the special case of $L=0, E=X \times \mathbb{C P}^{1}$, this gives the construction of a Lefschetz pencil. The $W$ in the lemma is the closure of the graph of a map from $X \backslash T$ to $\mathbb{C P}^{1}$.

Remark 4.2. The $T$ in the lemma is not generic, since it has to be the intersection of two hypersurfaces. For example, let $X=\mathbb{C P}^{2}, D=2 H$, $L=H$, then $T$ is 6 points and $W$ is $\mathbb{C P}^{2}$ blow-up $T$. But $T$ is not generic since the 6 points lie on a conic. Thus, $W$ is not a cubic surface. Instead, one can check that $W$ is the resolution of a cubic surface with an ordinary double point. (cf. [19] Ch 1, Ex 13)

Now fix some $k>0$ and assume that $k L+D$ is ample. Let $\mathcal{L}^{k}=\mathcal{L}^{\otimes k}$ is the $k$-th tensor power of $\mathcal{L}$ and $\mathcal{L}_{D}^{k}=\mathcal{L}^{k} \otimes \mathcal{D} \oplus \mathcal{D}$. Let $s_{D}^{\prime \prime}$ be a generic section of $\mathcal{L}^{k} \otimes \mathcal{D}$, then $s_{D}^{\prime \prime}$ defines a generic hypersurface $D_{0}^{\prime}$ in the linear

system $|k L+D|$ and $s_{k}=\frac{s_{D}^{\prime \prime}}{s_{D}}$ is a (meromorphic) section of $\mathcal{L}^{k}$. Define a "multi-section" $r$ of $\mathcal{L}$ from $s_{k}$ as:

$$
(r(p))^{\otimes k}=s_{k}(p) .
$$

Consider $r_{\lambda, D}=\left(\lambda_{1} r, \lambda_{0}\right)$. Let $W_{\lambda}^{k}$ be the closure of the image of $r_{\lambda, D}$ in $E$, then similar to (4.1), we have

$$
W_{\lambda}^{k} \cap V_{0}=D_{0}^{\prime}, \quad W_{\lambda}^{k} \cap V_{\infty}=D_{\infty}, \quad \text { and } W_{\lambda}^{k} \cdot f=k .
$$

This is very similar to the "Branched Covering Trick" (cf. [4]) in which one constructs a branched covering of $X$ with branching loci $B$ in $|k L|$ for some divisor $L$. Here, $W_{\lambda}^{k}$ is generically a branched $k$-ple covering of $X$ and contains the whole fiber $\mathbb{C P}^{1}$ over the intersection $D_{0}^{\prime} \cap D_{\infty}$. In fact, $W_{\lambda}^{k}$ can be defined by a generic section of the line bundle $\mathcal{O}_{E}(k) \otimes \pi^{*} \mathcal{D}$ over $E$. For $k=1$, this is the case of lemma 4.1 (cf. [9]§II.7).

\subsection{Semistable degeneration of algebraic surfaces.}

We consider here a generic element in the linear system $|d H|$ in $\mathbb{C P}^{3}$, i.e. a degree $d$ surface $S$. Let

$$
\triangle=\left\{\left(x_{1}, x_{2}, x_{3}\right) \mid x_{i} \geq 0, \text { for } i=1,2,3 \text { and } \sum_{i=1}^{3} x_{i} \leq d\right\} \subset \mathbb{R}^{3},
$$

then every nonsingular subdivision of $\triangle$ give raise to a semistable degeneration of $S$. We describe some obviously "universal" such subdivisions and the 
corresponding semistable degenerations. Then we'll treat specifically the degeneration of $K 3$ surfaces, since it leads to some interesting generalizations in higher dimensional Calabi-Yau hypersurfaces.

4.3.1. Degeneration into a chain of rational surfaces. 4.3.1.1. Consider the subdivision $\Gamma_{d}$ defined by the following hyperplanes:

$$
L_{j}=\left\{\left(x_{1}, x_{2}, x_{3}\right) \mid \sum_{i=1}^{3} x_{i}=j\right\}, \text { for } j=1, \ldots, d-1 .
$$

Label the subpolytope between $L_{j-1}$ and $L_{j}$ as $\triangle_{j}$ for $j=1, \ldots, d$. Let $Y_{j}$ be the toric variety defined by $\triangle_{j}$, then except that $Y_{1} \simeq \mathbb{C P}^{3}$, we have $Y_{j} \simeq \widetilde{\mathbb{C P}^{3}}$, the blowup of $\mathbb{C P}^{3}$ at one point, for $j=2, \ldots, d$. Let $H$ be the pullback of the hyperplane class and $E$ be class of the exceptional divisor, then they generate $H^{2}\left(\widetilde{\mathbb{C P}^{3}}\right)$. Let $D_{j}$ be the divisor on $Y_{j}$ defined by $\triangle_{j}$. Then

$$
D_{1}=H, \quad \text { and } \quad D_{j}=j H-(j-1) E \text { for } j \neq 1 .
$$

Note that $\widetilde{\mathbb{C P}^{3}} \simeq \mathbb{P}_{\mathbb{C P}^{2}}(\mathcal{O}(-1) \oplus \mathbf{1})$. Let $f$ denote the class of a fiber $\mathbb{C P}^{1}$, then we have the intersections

$$
H \cdot f=E \cdot f=1 \Rightarrow D_{j} \cdot f=j-(j-1)=1 .
$$

There are $d$ subpolytopes thus $S$ degenerates into $d$ components $S_{j}, j=$ $1, \ldots, d$. Each $S_{j}$ is a toric hypersurface of the corresponding $Y_{j}$ defined by $D_{j}$. The intersections $D_{j} \cdot H$ and $D_{j} \cdot E$ in $H \simeq E \simeq \mathbb{C P}^{2}$ are curves of degree $j$ and $j-1$ respectively. Thus, by lemma $4.1, X_{j}$ is the blow-up of $\mathbb{C P}^{2}$ at the intersection of degree $j$ and $j-1$ curves, which is $j(j-1)$ points. It's clear from the above description that the intersection of the components $X_{j}$ and $X_{j+1}$ is a degree $j$ curve, whose self-intersection number is $j$ in $X_{j}$, for all $0<j<d$.

4.3.1.2. More generally, consider the subdivision $\Gamma_{d}^{k}$ defined by the hyperplanes $L_{j}^{k}$ :

$$
L_{j}^{k}=\left\{\left(x_{1}, x_{2}, x_{3}\right) \mid \sum_{i=1}^{k} x_{i}=j\right\}, \quad \text { for } j=1, \ldots, d-1, \text { where } 1 \leq k \leq 3 .
$$

Notice that $k=1$ and $k=3$ give isomorphic subdivisions in the sense that one is transformed to the other by an integral affine isomorphism that maps 

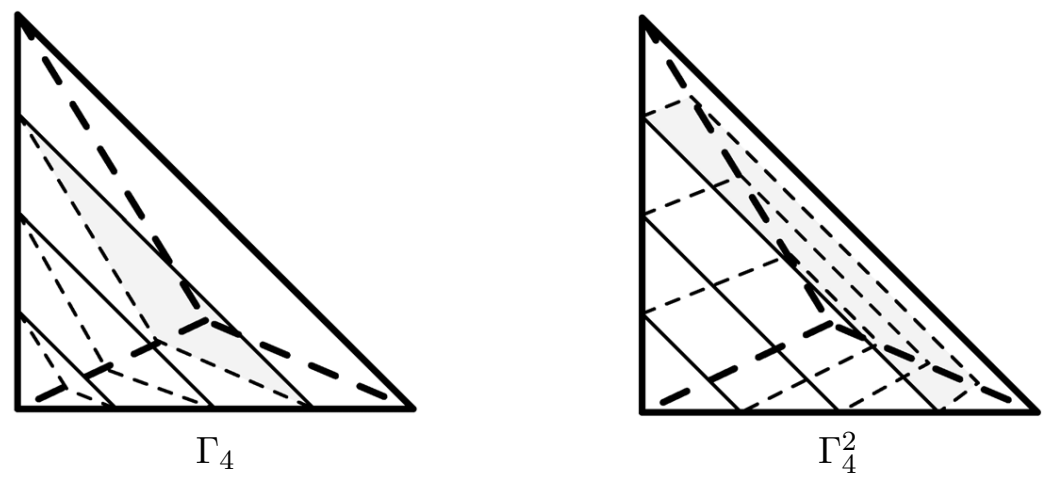

Figure 5 .

$\triangle_{d}$ to itself. Thus $k=2$ is the only new case. Figure 5 shows the two subdivisions for $d=4$.

We label the polytope between $L_{j-1}^{2}$ and $L_{j}^{2}$ as $\triangle_{j}^{2}$ and let $Y_{j}^{2}$ be the toric variety defined by $\triangle_{j}^{2}$, for $1 \leq j \leq d$. Then $Y_{j}^{2}$ is isomorphic to $\mathbb{C P}^{3}$ blow-up two non-intersecting line for $1<j<d$ and is isomorphic to $\mathbb{C P}^{3}$ blow-up a line for $j=1, d$. An equivalent description of the $Y_{j}^{2}$ 's is the following:

$$
\begin{aligned}
Y_{j}^{2} & \simeq Y^{2}=\mathbb{P}_{\mathbb{C P}^{1} \times \mathbb{C P}^{1}}\left(p_{1}^{*} \mathcal{O}(1) \otimes p_{2}^{*} \mathcal{O}(-1) \oplus \mathcal{O}\right), \text { for } 1<j<d, \\
\text { and } Y_{1}^{2} & \simeq Y_{d}^{2} \simeq Z=\mathbb{P}_{\mathbb{C P}^{1}}(\mathcal{O}(1) \oplus \mathcal{O}(1) \oplus \mathcal{O}),
\end{aligned}
$$

where $p_{1}\left(p_{2}\right)$ are projections to the first (resp. second) factor.

First consider $1<j<d$. Let $D_{0}$ (resp. $D_{\infty}$ ) denote the class of the divisor defined by the facet on $L_{j}^{2}$ (resp. $L_{j-1}^{2}$ ), then it's the class of the 0-section (resp. $\infty$-section) of the $\mathbb{C P}^{1}$ bundle. Then $\triangle_{j}^{2}$ defines the divisor:

$$
D_{j}^{2}=j D_{0}-(j-1) D_{\infty}+d H^{\prime}=(d+j-1) D_{\infty}-(d-j) D_{0}+d H^{\prime \prime},
$$

where $H=H^{\prime}+D_{0}=H^{\prime \prime}+D_{\infty}$ is the pullback of the hyperplane class in $\mathbb{C P}^{3}$ via the blow-down map. The component $X_{j}^{2}$ of the central fiber of the degeneration is a toric hypersurface of $Y_{j}^{2}$ in the linear system $\left|D_{j}^{2}\right|$. Let $f$ be the class of the fiber then we have the following:

$$
D_{0} \cdot f=D_{\infty} \cdot f=1 \text { and } H^{\prime} \cdot f=0 \Rightarrow D_{j}^{k} \cdot f=1,
$$

which shows that $X_{j}^{2}$ is generically a section. We compute

$$
\begin{aligned}
D_{j}^{2} \cdot D_{0} & =j H^{\prime \prime} \cdot D_{0}+(d-j) H^{\prime} \cdot D_{0} \\
D_{j}^{2} \cdot D_{\infty} & =(j-1) H^{\prime \prime} \cdot D_{\infty}+(d-j+1) H^{\prime} \cdot D_{\infty}
\end{aligned}
$$


Let $H_{1}=s^{*} H^{\prime \prime}$ and $H_{2}=s^{*} H^{\prime}$, where $s$ stands for 0 or $\infty$-section of the $\mathbb{C P}^{1}$ bundle, then $H_{i}$ is the divisor class of a fiber of $p_{i}$ in $\mathbb{C P}^{1} \times \mathbb{C P}^{1}$. It follows from lemma 4.1 that $X_{j}^{2}$ is the blown-up of $\mathbb{C P}^{1} \times \mathbb{C P}^{1}$ along the intersection:

$\left(j H_{1}+(d-j) H_{2}\right) \cdot\left((j-1) H_{1}+(d-j+1) H_{2}\right)=2 j(d-j+1)-d$ points.

It follows that the components $X_{j}^{2}$ and $X_{j+1}^{2}$ intersect along a curve of bidegree $(j, d-j)$, whose intersection number is $d-2 j$ in $X_{j}$ for $0<j<d$.

For $j=1, \triangle_{1}^{2}$ is shown in figure 6 . We label the facets as shown and the

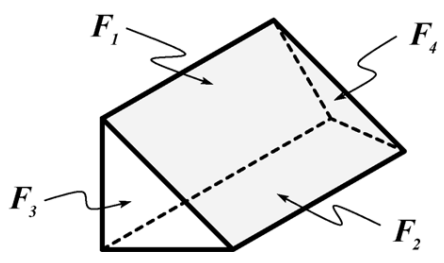

Figure 6.

shaded rectangular facet is labelled $F_{5}$. The divisors defined by the facets are also denoted $F_{i}$. In our notation, the class of the exceptional divisor is $F_{5}, F_{1}=F_{5}+F_{4}$ is the pullback of the hyperplane class from $\mathbb{C P}^{3}$ and the divisor defined by $\triangle_{1}^{2}$ is:

$$
D_{1}^{2}=F_{5}+d F_{4} .
$$

Then $X_{1}^{2}$ is a hypersurface in the linear system $\left|D_{1}^{2}\right|$.

Proposition 4.3. $X_{1}^{2}$ is in the list $\left\{\mathbb{F}_{d}, \mathbb{F}_{d-2}, \ldots\right\}$.

Proof: Let $\ell_{1}=F_{4} \cdot F_{5}, \ell_{2}=F_{5} \cdot F_{1}$. Then by simple calculation, we see that

$$
D_{1}^{2} \cdot F_{4}=\ell_{1}, \quad D_{1}^{2} \cdot F_{5}=(d-1) \ell_{1}+\ell_{2} \quad \text { and } \quad D_{1}^{2} \cdot F_{1}=d \ell_{1}+\ell_{2} .
$$

The first equation tells us that $X_{1}^{2}$ is a $\mathbb{C P}^{1}$ bundle over $\mathbb{C P}^{1}$. The last two intersections are the sections of this $\mathbb{C P}^{1}$ bundle. Calculating the intersection numbers of the two sections, we see that $X_{1}^{2}$ is a Hirtzebruch surface in the list $\left\{\mathbb{F}_{d+2 i}, \quad i \in \mathbb{Z}\right\}$. We need more information to pin down the $i$, which can be seen in the following way of determining $X_{1}^{2}$.

Consider the divisor $D_{1}^{2}=F_{5}+d F_{4}$ in $Y^{2}$, then a generic hypersurface $\widetilde{X_{1}^{2}}$ in $\left|D_{1}^{2}\right|$ is generically a section. By lemma 4.1 , we see that $\widetilde{X_{1}^{2}}$ is a blow-up 
of $\mathbb{C P}^{1} \times \mathbb{C P}^{1}$ at $\left(H_{1}+(d-1) H_{2}\right) \cdot d H_{2}=d$ points, where $H_{i}$ is the divisor class of the fiber of projection to the $i$-th factor. Let $\ell=\left|\triangle_{1}^{2} \cap \mathbb{Z}^{n}\right|$, then morphism $\phi_{1}^{2}$ of $Y^{2}$ to $\mathbb{C P}^{\ell-1}$ defined by $\triangle_{1}^{2}$ contracts the fiber of $p_{2}$ in $F$, from which $Y_{1}^{2}$ appears as the image. The image of generic $\widetilde{X_{1}^{2}}$ is a (smooth) hypersurface of $Y_{1}^{2}$, which is $X_{1}^{2}$. Since the map $Y^{2} \rightarrow Y_{1}^{2}$ is a blow-up, we see that $\widetilde{X_{1}^{2}} \rightarrow X_{1}^{2}$ is a blow-up as well. In fact, since $D_{1}^{2} \cdot D_{\infty}=d H_{2}$, $\widetilde{X_{1}^{2}}$ is the blow-up of $X_{1}^{2}$ at $d$ points. Thus, $X_{1}^{2}$ is rational and fits into the blowing-up diagram

$$
\mathbb{C P}^{1} \times \mathbb{C P}^{1} \leftarrow \widetilde{X_{1}^{2}} \rightarrow X_{1}^{2} .
$$

In general, the blowing-up blowing-down process as above can only tell us that $X_{1}^{2}$ is in the list $\left\{\mathbb{F}_{d}, \mathbb{F}_{d-2}, \ldots\right\}$.

Similar arguments as above apply for $j=d$. Thus, the subdivision $\Gamma^{2}$ gives rise to a semistable degeneration of degree $d$ surface into a chain of rational surfaces, with both ends in the list $\left\{\mathbb{F}_{d}, \mathbb{F}_{d-2}, \ldots\right\}$.

4.3.2. Inductive structure. We can subdivision the polytope $\triangle_{d}$ using some of the hyperplanes $L_{j}$ as above and get a subdivision which has fewer subpolytopes. One special case is to take the subdivision of $\triangle_{d}$ given by a single hyperplane $L_{d-1}$. We end up with a semistable degeneration of degree $d$ surface into two components. One of the component is a degree $d-1$ surface and the other is the $X_{d}$ described above, which is the blow-up of $\mathbb{C P}^{2}$ at $d(d-1)$ points. Thus, surfaces of all degrees in $\mathbb{C P}^{3}$ as a system has an "inductive" structure.

Remark 4.4. The degenerations described in these two subsections easily generalize to higher dimension and leads to similar structures in higher dimensional hypersurfaces.

4.3.3. Degeneration of $K 3$ surfaces. 4.3.3.1. Consider the subdivision of $\triangle_{4}$ into 2 pieces by $L_{2}^{2}$ and denote the resulting toric varieties as $Z_{1}$ and $Z_{2}$. Then $Z_{1} \simeq Z_{2} \simeq Z$ as in (4.4). The subpolytopes define the same divisor in $Z$ :

$$
D=2 F_{5}+4 F_{4} .
$$

This subdivision induces a semistable degeneration of $K 3$ surface into 2 components $P_{i}, i=1,2$. Then $P_{i}$ are in the same linear system $|D|$, thus $P_{1} \simeq P_{2} \simeq P$.

Proposition 4.5. $P \simeq E(1)$ as topological manifolds. 
Proof: It's easy to compute the intersection $D \cdot F_{5}=2 \ell_{1}+2 \ell_{2}$, then by genus formula we see that $P$ intersects with $F_{5}$ in an elliptic curve $A$. By adjunction

$$
K_{P}=\left.\left(K_{Z}+D\right)\right|_{P}=\left.\left(-F_{5}\right)\right|_{P},
$$

we see that $K_{P}=-A$ and

$$
c_{1}^{2}(P)=A \cdot A=\left.\left(F_{5}\right)^{2}\right|_{P}=\left(F_{5}\right)^{2} \cdot D=0 .
$$

By Lefschetz hypersurface theorem, we have $q(P)=0$. Since $-K_{P}=A$ is effective, $K_{P} \cdot K_{P}=0$ and $q(P)=0$, we see that $P$ is rational by the Castelnuovo-Enriques theorem. Using the cohomology sequence associated to the exact sequence

$$
0 \rightarrow \mathcal{O}_{Z}(-D) \rightarrow \mathcal{O}_{Z} \rightarrow \mathcal{O}_{P} \rightarrow 0
$$

and $h^{2}\left(\mathcal{O}_{Z}\right)=h^{3}\left(\mathcal{O}_{Z}\right)=0$, we see that

$$
h^{2}\left(\mathcal{O}_{P}\right)=h^{3}\left(\mathcal{O}_{Z}(-D)\right)=0,
$$

while the latter is 0 by Serre duality and Kodaira vanishing. By Noether's formula, we get $e(P)=c_{2}(P)=12$ and thus $h^{2}(P)=h^{1,1}(P)=10$. Then the signature

$$
\sigma(P)=\frac{1}{3}\left(c_{1}^{2}(P)-2 c_{2}(P)\right)=-8 .
$$

Let $Q$ be the intersection form of $P$, then $Q=\langle 1\rangle \oplus 9\langle-1\rangle$ if it's odd, or $Q=-E_{8} \oplus H$ if it's even where $H$ is the intersection form of 2-torus. From 4-manifold theory, $Q \neq-E_{8} \oplus H$, which gives

$$
Q=\langle 1\rangle \oplus 9\langle-1\rangle \text {. }
$$

We see that $P$ is the blow-up of some ruled surface $\mathbb{F}_{q}$ at 8 points. Thus, topologically, we have $P \simeq E(1)$.

In light of proposition 4.5, we may think of the degeneration as an algebraic geometrical version of the fiber sum operation.

4.3.3.2. Consider the subdivision defined by $L_{3}^{2}$. There are two components in the central fiber of the induced semistable degeneration of $K 3$ surfaces. Both components are hypersurfaces in $Z$. One of them, say $W_{1}$, is in the linear system $\left|F_{5}+4 F_{4}\right|$, which by proposition 4.3 is one of $\mathbb{F}_{4}, \mathbb{F}_{2}$ and $\mathbb{C P}^{1} \times$ $\mathbb{C P}^{1}$. The other, $W_{2}$, is in the linear system $\left|3 F_{5}+4 F_{4}\right|$. By toric geometry, the canonical class of $Z$ is

$$
K_{Z}=-\sum_{i=1}^{5} F_{5}=-\left(3 F_{5}+4 F_{4}\right) .
$$


Thus, $K_{W_{2}}=\left.\left(W_{2}+K_{Z}\right)\right|_{W_{2}}=0$ by adjunction and $h^{1}\left(W_{2}\right)=0$ by Lefschetz hypersurface theorem. We see that $W_{2}$ is again a $K 3$ surface. The two components intersect along a rational curve $\ell$ by the calculation in the first subsection and the self-intersection of $\ell$ in $W_{2}$ is -2 either by checking directly or using genus formula.

\subsection{Semistable degeneration of Calabi-Yau hypersurfaces.}

4.4.1. Classical degeneration of $K 3$ and its generalizations. We'll use the notations of example 3.8. Recall that $Q^{n}$ is defined by the polytope $\triangle_{0}(n) \subset \mathbb{R}^{n}$. The 1-skeleton of the normal fan $\Sigma_{0}(n)$ of $\triangle_{0}(n)$ is generated by $2 n$ vectors:

$$
e_{1}, \ldots, e_{n} \text { and } w_{j}=-\sum_{i=1}^{j} e_{i} \text { for } j=1, \ldots, n \text {. }
$$

Let $D_{1}, \ldots, D_{n}$ and $D_{n+1}, \ldots, D_{2 n}$ be the divisors corresponding to $e_{1}, \ldots, e_{n}$ and $w_{1}, \ldots, w_{n}$ respectively. Then $\triangle_{0}(n)$ defines the divisor $D$ on $Q^{n}$ :

$$
D=\sum_{i=1}^{n} D_{i}
$$

Sometimes we will use $D_{i}(n)$ to emphasize that $D_{i}$ is a divisor of $Q^{n}$. In the description of $Q^{n}$ as $\mathbb{C P}^{n}$ blown-up a complete flag, $D_{1}(n)$ is the pull-back of the hyperplane class on $\mathbb{C P}^{n}$. The properties of $Q^{n}$ are collected in the following:

Proposition 4.6. Fix any $n>0$ and set $Q^{0}=p t$, we have the following:

1. $Q^{n}$ is rational.

2. $Q^{n}$ is Fano, with canonical class $K=-\sum_{i=1}^{2 n} D_{i}$.

3. $D_{j}=\sum_{i=j}^{n} D_{n+i}$ for $j=1, \ldots, n$.

4. $Q^{n}=\mathbb{P}_{Q^{n-1}}\left(\mathcal{D}_{1}(n-1) \oplus \mathbf{1}\right)$.

5. $D_{1}$ and $D_{n+1}$ are (resp. 0 - and $\infty$-) sections of the above bundle.

6. Let $f$ be the class of the fiber, then $D_{i} \cdot f=0$ when $i \neq 1, n+1$.

7. $\left.D_{1}(n)\right|_{D_{1}}=D_{1}(n-1)$ and $\left.D_{n+1}(n)\right|_{D_{n+1}}=-D_{1}(n-1)$, 
8. Let $\pi_{n}: Q^{n} \rightarrow Q^{n-1}$ be the bundle map, then $D_{i+1}(n)=\pi_{n}^{-1}\left(D_{i}(n-\right.$ $1)$ ), for $i=1, \ldots, n-1$ and $D_{i+2}(n)=\pi_{n}^{-1}\left(D_{i}(n-1)\right)$, for $i=$ $n, \ldots, 2(n-1)$.

9. $h^{p, p}\left(Q^{n}\right)=C_{n}^{p}$ for all $p$.

Now we should describe the degeneration of a Calabi-Yau hypersurface $V$ in $\mathbb{C P}^{n}$. The components in the central fiber of the degeneration of $V$ will consists of $n+1$ hypersurfaces of the Fano variety $Q^{n}$ in the linear system $|D|$. Let $C^{n-1}$ be a generic element in $|D|$ then $C^{n-1}$ is generically a section of the $\mathbb{C P}^{1}$ bundle $Q^{n} \rightarrow Q^{n-1}$. It then follows from lemma 4.1 that $C^{n-1}$ rational. It's easy to see from the subdivision $\Gamma$ that the dual graph of the central fiber $\left(V_{0}\right)$ is a triangulation of $S^{n-1}$ by $n+1$ simplices. To find more information on $C^{n-1}$ we compute the intersections of $D$ with the 0 - and $\infty$-sections.

4.4.1.1. $n=3$, i.e. $K 3$ surfaces in $\mathbb{C P}^{3}$. Recall $Q^{2}=\widetilde{\mathbb{C P}^{2}}$ with exceptional divisor $E=D_{3}(2)$ and $Q^{3}=\mathbb{P}_{\widetilde{\mathbb{C P}^{2}}}(\mathcal{H} \oplus \mathbf{1})$ where $H=D_{1}(2)$. Then

$$
D \cdot D_{1}=2 H+f \text { and } D \cdot D_{4}=H+f
$$

as divisor in $\widetilde{\mathbb{C P}^{2}}$. Note that $2 H+f$ is the anticanonical divisor of $\widetilde{\mathbb{C P}^{2}}$. Thus, $C^{2}$ is $\widetilde{\mathbb{C P}^{2}}$ blow-up

$$
(2 H+f) \cdot(H+f)=5 \text { points. }
$$

Contracting $E$, we see that $C^{2}$ is isomorphic to $\mathbb{C P}^{2}$ blown-up the intersection of two curves of degree 2 and 3 . As in remark 4.2, we get the resolution of a cubic hypersurface with an ordinary double point.

Since the $Q^{3}$ 's in the central fiber of the degeneration of $\mathbb{C P}^{3}$ meet along the subvarieties $D_{4}, D_{5}$ and $D_{6}$, we can get the singular loci of $V_{0}$ by the following computation:

$D_{4}: D \cdot D_{4}=H+f$, we see that the intersection is a $\mathbb{C P}^{1}$.

$D_{5}$ : is also isomorphic to $\widetilde{\mathbb{C P}^{2}}$, then $D \cdot D_{5}=H+f$ gives a $\mathbb{C P}^{1}$ as well.

$D_{6}$ : is isomorphic to $\mathbb{C P}^{1} \times \mathbb{C P}^{1}$ and $D \cdot D_{6}$ is of bidegree $(1,1)$ thus we get another $\mathbb{C P}^{1}$. 
Thus, the components of $V_{0}$ intersect along $\mathbb{C P}^{1}$ 's and we recover the known construction of $K 3$ degenerates into 4 rational surfaces.

4.4.1.2. $n=4$, i.e. quintics in $\mathbb{C P}^{4}$. In the $\mathbb{C P}^{1}$-bundle $Q^{4}=$ $\mathbb{P}_{Q^{3}}\left(D_{1}(3) \oplus \mathbf{1}\right) \rightarrow Q^{3}, D_{1}$ is the 0 -section and $D_{5}$ is the $\infty$-section. Let $K(3)$ be the canonical class of $Q^{3}$ then we have the following intersections:

$$
\begin{gathered}
D \cdot D_{1}=\left(\sum_{i=2}^{8} D_{i}\right) \cdot D_{1}=-K(3), \\
D \cdot D_{5}=-K(3)-D_{1}(3)=\sum_{i=1}^{3} D_{i}(3) .
\end{gathered}
$$

Thus $C^{3}$ is isomorphic to $Q^{3}$ blown-up along the intersection of a $K 3$ surface and $C^{2}$ in $Q^{3}$. One can check that the curve $T$ has genus 12 and is in the class $8 D_{1}(2)-3 D_{3}(2)+3 f$ where $D_{1}(2)$ (resp. $\left.D_{3}(2)\right)$ is the image of the divisor $D_{1}$ (resp. $D_{3}$ ) in $Q^{2}$ under the embedding $Q^{2}=D_{1}(3) \hookrightarrow Q^{3}=D_{1}(4) \hookrightarrow Q^{4}$, while $f$ is the class of fiber in $Q^{4}$.

4.4.1.3. In general, $C^{n}$ is a blow-up of $Q^{n}$ along the intersection of two hypersurfaces in the linear systems $\left|\sum_{i=1}^{2 n} D_{i}(n)\right|$ and $\left|\sum_{i=1}^{n} D_{i}(n)\right|$ respectively. The first one is a Calabi-Yau since it's in the anticanonical class and the second one is in fact $C^{n-1}$. The class of the intersection can be computed as following. Let $D_{\overline{1}}(n)=\sum_{i=2}^{n} D_{i}(n)$, then

$$
D_{\overline{1}}(n)=\pi_{n}^{-1}\left(\sum_{i=1}^{n-1} D_{i}(n-1)\right)=\pi_{n}^{-1}\left(C^{n-1}\right) .
$$

Since $\sum_{i=1}^{2 n} D_{i}(n)=2 D_{1}(n)+D_{\overline{1}}$, we have

$$
\begin{aligned}
T & =\sum_{i=1}^{2 n} D_{i}(n) \cdot \sum_{i=1}^{n} D_{i}(n) \\
& =2 D_{1}(n) \cdot D_{1}(n)+3 D_{1}(n) \cdot D_{\overline{1}}(n)+D_{\overline{1}}(n) \cdot D_{\overline{1}}(n) \\
& =2 D_{1}(n-1)+3 C^{n-1}+\pi_{n}^{-1}\left(C^{n-1} \cdot C^{n-1}\right) .
\end{aligned}
$$

where $D_{1}(n-1)$ and $C^{n-1}$ are regarded as classes of $Q^{n}$ by the embedding $Q^{n-1}=D_{1}(n) \hookrightarrow Q^{n}$. The computation for $C^{n-1} \cdot C^{n-1}$ can be carried out similarly and eventually we arrive at an expression involving $D_{1}(j), C^{j}$ and composition of pull-backs by $\pi_{j}$.

We summarize what we got as the following: 
Theorem 4.7. There exists a semistable degeneration of degree $n+1$ Calabi-Yau hypersurface in $\mathbb{C P}^{n}$, such that the dual graph of the central fiber is the triangulation of $S^{n-1}$ by the boundary of n-simplex. The components in the central fiber are all isomorphic to a blow-up of $Q^{n-1}$ along the intersection of two hypersurfaces.

4.4.2. Degeneration of Calabi-Yau hypersurfaces in $\mathbb{C P}^{2 k+1}$. In this subsection, we generalize the degeneration of $K 3$ surface into two copies of $E(1)$. Although it's hard to determine the components in the central fiber, we find that the many properties generalize.

We consider the subdivision of $\triangle_{2 k+2}$ defined by $L_{k+1}^{k+1}$ and denote the resulting toric varieties as $X_{1}$ and $X_{2}$. It's easy to check that

$$
X_{1} \simeq X_{2} \simeq X=\text { the blow-up of } \mathbb{C P}^{2 k+1} \text { along linear subspace } \mathbb{C P}^{k} \text {. }
$$

Equivalently, we have $X=\mathbb{P}_{\mathbb{C P}^{k}}\left(\mathcal{O}^{k+1}(1) \oplus \mathbf{1}\right)$. The exceptional divisor is $E=\mathbb{C P}^{k} \times \mathbb{C P}^{k}$ and the canonical class of $E$ is $K_{E}=-(k+1)\left(p_{1}^{*} H_{1}+p_{2}^{*} H_{2}\right)$, where $p_{i}$ (resp. $H_{i}$ ) is the projections to (resp. hyperplane class on) the $i$-th factor $(i=1,2)$. Let $H=H^{\prime}+E$ be the pull back of the hyperplane class in $\mathbb{C P}^{2 k+1}$, then simple calculation gives:

$K_{X}=-(2 k+2) H^{\prime}-(k+2) E$, and $E \cdot E=\left(H-H^{\prime}\right) \cdot E=p_{1}^{*} H_{1}-p_{2}^{*} H_{2}$,

where $p_{i}^{*} H_{i}$ is regarded as classes in $X$ via the inclusion $E \hookrightarrow X$.

The induced semistable degeneration of Calabi-Yau hypersurface has two components in the central fiber, say $Y_{i} \subset X_{i}(i=1,2)$. The divisors defined by $\triangle_{i}(i=1,2)$ are both linearly equivalent to $D=(k+1)\left(H^{\prime}+H\right)$. It follows that $Y_{1} \simeq Y_{2} \simeq Y$ and the canonical class of $Y$ is $K_{Y}=\left.(-E)\right|_{Y}$. By $\left.D\right|_{E}=\left.(k+1)\left(H^{\prime}+H\right)\right|_{E}=(k+1)\left(p_{1}^{*} H_{1}+p_{2}^{*} H_{2}\right)=-K_{E}$, we see that the anticanonical class $-K_{Y}$ defines a $2 k-1$ dimensional Calabi-Yau $Z$ in $Y$. The two components $Y_{i}$ intersect along $Z$. By property of semistable degeneration, the self-intersection of $Z$ in the components add up to 0 , while $Z$ has same self-intersection in this case, we see that $Z \cdot Z=0$, i.e. $\left(K_{Y}\right)^{2}=0$. We can state the result as:

Theorem 4.8. There exists a semistable degeneration of Calabi-Yau hypersurface in $\mathbb{C P}^{2 k+1}$ into two components $Y_{i}(i=1,2)$, such that $Y_{1} \simeq Y_{2} \simeq Y$ satisfy the following: $\left(K_{Y}\right)^{2}=0,-K_{Y}$ is effective and defines a Calabi-Yau subvariety $Z$ of $Y, Z$ is isomorphic to a hypersurface in $\mathbb{C P}^{k} \times \mathbb{C P}^{k}$ and the singular loci of the central fiber is $Z$. 
4.4.3. Degeneration of Calabi-Yau hypersurface in $\mathbb{P}(1,1,2,2,2)$. By theorem 3.5 and example 3.9, we obtain a weak semistable degeneration of the Calabi-Yau hypersurface in $\mathbb{P}(1,1,2,2,2)$ into 5 components. There is one smooth component which is isomorphic to $C^{4}$ in $\S 4.4 .1$. The other four components are toric hypersurfaces with quotient singularities.

\section{Higher dimensional base.}

We can construct higher dimensional liftings for certain subdivisions, which leads to degeneration with higher dimensional base. For simplicity, we'll assume that polytopes and subdivisions are nonsingular.

Suppose $\Gamma$ is a subdivision of polytope $\triangle$ with $\operatorname{dim} K_{\Gamma}=1$, then $\triangle$ is subdivided into a chain of subpolytopes $\left\{\triangle_{i}\right\}_{i=0}^{l}$. Let the dividing hyperplane $L_{i}$ be defined by $\left\{\left\langle m, n_{i}\right\rangle=c_{i}\right\}$ where $n_{i} \in N$ is primary vector for $i=1, \ldots, l$. We perform the lifting in $l$ steps as following.

Step 1: Lift $\triangle$ by the subdivision defined by $L_{1}$, to $\triangle_{1} \subset \triangle \times \mathbb{R} \subset$ $M_{\mathbb{R}} \times \mathbb{R}$. Let $L_{i ; 1}$ be the preimage of $L_{i}(i>1)$ under the projection $\pi_{1}: M_{\mathbb{R}} \times \mathbb{R} \rightarrow M_{\mathbb{R}}$, then $L_{i ; 1}(i>1)$ define a subdivision $\Gamma_{1}$ of $\triangle_{1}$. It's easy to check that $\Gamma_{1}$ is nonsingular, given everything we started with is nonsingular.

Step 2: Repeat Step 1 for $\triangle_{1}, \Gamma_{1}$ and $L_{2 ; 1}$ to get $\triangle_{2} \subset M_{\mathbb{R}} \times \mathbb{R}^{2}$ and subdivision $\Gamma_{2}$ of $\triangle_{2}$ given by $(l-2)$ hyperplanes $L_{i ; 2}(i>2)$ which are the preimage of $L_{i}(i>2)$ under the projection $\pi_{2}: M_{\mathbb{R}} \times \mathbb{R}^{2} \rightarrow M_{\mathbb{R}}$.

Step $l$ : The result is a nonsingular polytope $\triangle_{l} \subset M_{\mathbb{R}} \times \mathbb{R}^{l}$.

In each step above, the lifting polytope $\triangle_{i}$ can be chosen to be either compact or open. The results of the first 2 steps for the subdivision on $[0,4]$ defined by lattice points $1,2,3$ is shown in figure 7 .

Another way to get the final result in one step is to construct a suitable function $F_{\Gamma, l}: \triangle \rightarrow \mathbb{R}^{l}$ and let $G_{\Gamma, l}$ be the graph of $F_{\Gamma, l}$ in $\triangle \times \mathbb{R}^{l}$. Then we get polytope $\triangle_{l}$ by taking the Minkowski sum of $G_{\Gamma, l}$ with $\left(\mathbb{R}^{+}\right)^{l}$. The function $F_{\Gamma, l}$ can be constructed in steps that are parallel to the steps above. Instead, we'll define it as following. Let $\left.\left\{e_{i}\right\}\right|_{i=1} ^{l}$ be the standard basis of $\mathbb{R}^{l}$. For each $L_{i}$, define $f_{i}=\left\langle m, n_{i}\right\rangle-c_{i}$, then $\left.f_{i}\right|_{L_{i}}=0$. Let $f_{0}=0$. Define

$$
F_{\Gamma, l}=\sum_{j=0}^{i} f_{i} e_{i}, \quad \text { on } \triangle_{i} \text { for } i=0, \ldots, l,
$$

then it's easy to check that $F_{\Gamma, l}$ induces the polytope $\triangle_{l}$. 


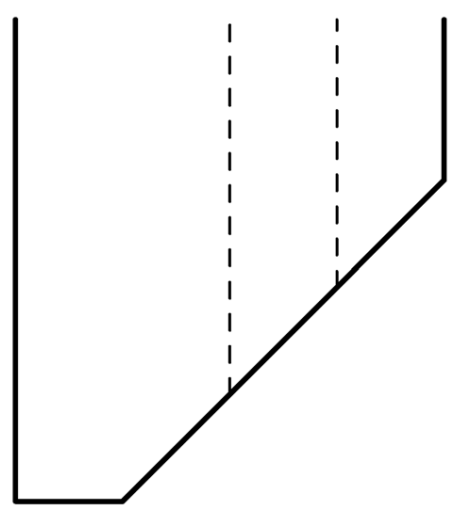

Step 1

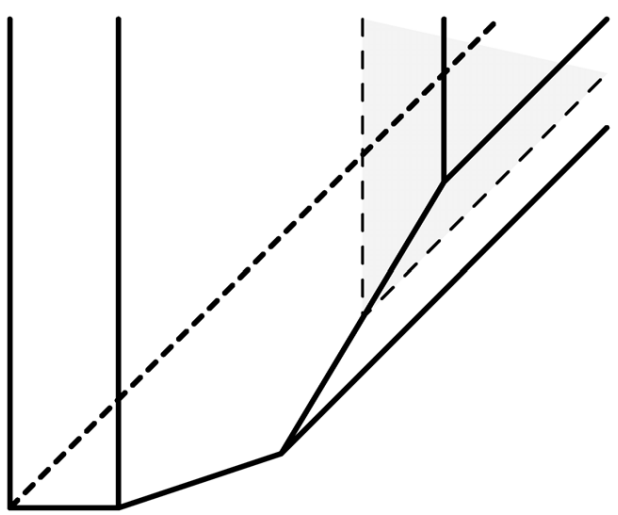

Step 2

Figure 7.

Example 5.1. Let $\triangle=\mathbb{R}$ the toric variety is then $\mathbb{C}^{\times}$. Without loss of generality, we let $\Gamma$ be defined by the integers $\{j \in \mathbb{Z} \mid 0<j<l\}$. The above construction recovers the construction of the special standard model of expanded degeneration in [16].

Remark 5.2. We note that in dimension 1 the subdivisions of $(\triangle, \triangle \cap M)$ defined by the lattice points are all semistable and nonsingular. Let $\triangle=[0, \ell]$ then the secondary polytope is combinatorially a cube of dimension $\ell-1$ (cf. [8]). The subdivisions defined by lattice points correspond not only to the facet of secondary polytope, but also the lower dimensional faces as well. In fact, the codimension of the face is the number of subdivision points. Restricting example 5.1 to finite interval $[0, \ldots, \ell]$, i.e. twisted rational curve in $\mathbb{C P}^{\ell}$, the construction gives explicit presentation of the Chow family over an affine piece of the Chow quotient $\mathbb{C P}^{\ell} / /{ }_{c h} \mathbb{C}^{\times}$containing the point corresponding to the finest subdivision $[0,1] \cup \ldots \cup[\ell-1, \ell]$.

Let $X$ (resp. $\left.\tilde{X}_{l}\right)$ be the toric variety defined by $\triangle\left(\right.$ resp. $\left.\triangle_{l}\right)$. By similar argument as in the proof of theorem 3.1, there is a morphism of toric varieties $p_{l}: \widetilde{X}_{l} \rightarrow \mathbb{C}^{l}$, with generic fiber $X$ and singular fibers over the coordinate planes in $\mathbb{C}^{l}$. The singular fibers correspond to subdivisions defined by subsets of $\left\{L_{i}\right\}_{i=1}^{l}$, especially, the fiber over $(0, \ldots, 0)$ corresponds to the original subdivision $\Gamma$. Again, we want to consider the induced degeneration of subvarieties of $X$. We'll consider compact liftings in the following and use the basic construction with compact polytopes. 
Lemma 5.3. Suppose $\triangle \subset M_{\mathbb{R}}$ is a nonsingular polytope and $\Gamma$ is a subdivision defined by a single hyperplane. Let $\widetilde{\triangle} \subset M_{\mathbb{R}} \times \mathbb{R}$ be a compact lifting of $\triangle$ by $\Gamma$. Let $\Sigma$ and $\widetilde{\Sigma}$ be the normal fans of $\triangle$ and $\widetilde{\triangle}$ respectively, then $\Sigma$ is a subfan of $\widetilde{\Sigma}$. Suppose $\phi$ is an integral convex piecewise linear function on $\Sigma$, then there exists an integral convex piecewise linear function $\widetilde{\phi}$ on $\widetilde{\Sigma}$ that restrict to $\phi$ on $\Sigma$.

Proof: By construction, $\widetilde{\Sigma}$ has three more 1-dimensional cones than $\Sigma$. We may assume that $-e_{1}=(0, \ldots, 0,-1) \in N_{\mathbb{R}} \times \mathbb{R}$ generates an edge of $\widetilde{\Sigma}$ and let $v_{1}$ and $v_{2}$ generate the two edges in upper half space. Let $\widetilde{\Sigma}_{i}$ be the fan generated by $\Sigma$ and $v_{i}$ for $i=1,2$, then $\left|\Sigma_{i}\right|=\overline{\mathbf{H}^{+}}=N_{\mathbb{R}} \times \overline{\mathbb{R}^{+}}$. Let $\pi_{i}: N_{\mathbb{R}} \times \mathbb{R} \rightarrow N_{\mathbb{R}}$ be the projection with kernel $\operatorname{Span}\left(v_{i}\right)$, then the function $\phi_{i}=\pi_{i}^{*}(\phi)$ is convex on the fan generated by $\Sigma$ and $v_{i}$, for $i=1,2$. By definition, $\phi_{1}\left(v_{1}\right)=\phi_{2}\left(v_{2}\right)=0$. Let

$$
\widetilde{\phi}=\max _{t \in[0,1]}\left(t \phi_{1}+(1-t) \phi_{2}\right),
$$

then the set $\{(x, y) \mid y \leq \widetilde{\phi}(x)\} \subset \overline{\mathbf{H}^{+}} \times \mathbb{R}$ is the convex hull of the corresponding sets of $\phi_{1}$ and $\phi_{2}$. Thus $\widetilde{\phi}$ is convex on $\overline{\mathbf{H}^{+}}$. Now, pick $0 \gg a \in \mathbb{Z}$ and set $\widetilde{\phi}\left(-e_{1}\right)=a$ and linearly extend to all $\mathbf{H}^{-}$on each cone of $\widetilde{\Sigma}$. Then $\widetilde{\phi}$ is convex on the whole $N_{\mathbb{R}} \times \mathbb{R}$. It's obvious that $\widetilde{\phi}$ is piecewise linear on $\widetilde{\Sigma}$ and integrality follows.

Remark 5.4. The function $\widetilde{\phi}$ constructed in the above proof has an extra feature, i.e. $\widetilde{\phi}=0$ on the cone generated by the new edges in $\widetilde{\Sigma}$.

Let $D=\sum_{\sigma} a_{\sigma} D_{\sigma}$ be a Cartier divisor on $X$, where $D_{\sigma}$ is the divisor corresponding to the facet $\sigma \prec \triangle$. The supporting function $\phi_{D}$ of $D$ is defined on the fan $\Sigma_{\triangle}$ by setting $\phi_{D}\left(v_{\sigma}\right)=-a_{\sigma}$ where $v_{\sigma}$ is the primary normal vector of $\sigma$ and extend so that $\phi_{D}$ is linear on each cone of $\Sigma_{\triangle}$. Suppose $D$ is generated by sections, then its support function $\phi_{D}$ is a convex integral function on $\Sigma$. Apply lemma 5.3 on $\phi_{D}$ inductively to each step of the (compact) lifting of $\triangle$ to $\triangle_{l}$, we get a function $\phi_{l}$ which is integral and convex on the fan $\widetilde{\Sigma}_{l}$ given by the compact lifting $\triangle_{l}$. Let $\widetilde{\sigma} \prec \triangle_{l}$ be the facet that maps to the facet $\sigma \prec \triangle$ under projection $N_{\mathbb{R}} \times \mathbb{R}^{l} \rightarrow N_{\mathbb{R}}$. By remark 5.4, we see that the divisor defined by $\phi_{l}$ is $\widetilde{D}_{l}=\sum_{\sigma} a_{\sigma} D_{\widetilde{\sigma}}$, where $D_{\widetilde{\sigma}}$ is the divisor on $\widetilde{X}_{l}$ defined by $\widetilde{\sigma}$. Since $\phi_{l}$ is convex, the linear system $\left|\widetilde{D}_{l}\right|$ is generated by sections. Let $V$ be a generic hypersurface of $X$ in the linear system $|D|$ and $\widetilde{V}$ be a generic hypersurface of $\widetilde{X}_{l}$ in the linear system $\left|\widetilde{D}_{l}\right|$, then $\widetilde{V}$ intersects the invariant divisors transversely. Thus, over 
a neighbourhood of $(0, \ldots, 0) \in \mathbb{C}^{n}$, we can restrict $p_{l}$ to $\widetilde{V}$ and obtain a semistable degeneration of $V$ over a $l$-dimensional base.

\section{References.}

[1] V. Alexeev, Complete Moduli in the Presence of Semiabelian Group Action, Ann. of Math. (2) 155 (2002), no. 3, 611-708; MR1923963 (2003g:14059).

[2] D. Ambramovich and K. Karu, Weak Semistable Reduction In Characteristic 0, Invent. Math. 139 (2000), no. 2, 241-273; MR1738451 (2001f:14021).

[3] L. J. Billera and B. Sturmfels, Fiber polytopes, Ann. of Maths, 2nd Ser. 135 (1992), no. 3, 527-549.

[4] W. Barth, A. Van de Ven and C.A.M. Peters, Compact complex surfaces, Ergebnisse der Mathematik und ihrer Grenzgebiete. 3. Folge, Bd. 4, Springer-Verlag, Berlin, 1984; MR0749574 (86c:32026).

[5] D. Cox, Recent Developements in Toric Geometry in Algebraic geometry - Santa Cruz 1995, 389-436, Proc. Sympos. Pure Math., Part 2, Amer. Math. Soc., Providence, RI, 1997; MR1492541 (99d:14054).

[6] D. Eisenbud and J. Harris, The Geometry of Schemes, Graduate Texts in Mathematics 197, Springer, New York, 2000; MR1730819 (2001d:14002).

[7] W. Fulton, Introduction to Toric Varieties, Annals of Mathematics Studies 131, Princeton University Press, 1993.

[8] I. M. Gelfand, M. M. Kapranov and A. V. Zelevinsky, Discriminants, Resultants and Multidimensional Determinants, Mathematics: Theory \& Applications, Birkhäuser, 1994.

[9] R. Hartshorne, Algebraic Geometry, Graduate Texts in Mathematics 52, Springer-Verlag, New York, 1977; MR0463157 (57 \#3116)

[10] Y. Hu, Topological Aspects of Chow Quotients, J. Diff. Geom. 69 (2005), no. $3,399-440$.

[11] E.-N. Ionel and T.H. Parker, The Symplectic Sum Formula for GromovWitten Invariants, Ann. Math. 159 (2004), no. 3, 935-1025. 
[12] G. Kempf, F. Knudsen, D. Mumford and B. Saint-Donat, Toroidal Embeddings I, Lecture Notes in Mathematics 339, Springer-Verlag, 1973.

[13] M. M. Kapranov, B. Sturmfels and A. V. Zelevinsky, Quotients of toric varieties, Math. Ann. 290 (1991), no. 4, 643-655; MR1119943 (92g:14050).

[14] M. M. Kapranov, B. Sturmfels and A. V. Zelevinsky, Chow polytopes and general resultants, Duke Math. J. 67 (1992), no. 1, 189-218.

[15] A.-M. Li and Y. Ruan, Symplectic surgery and Gromov-Witten invariants of Calabi-Yau 3-folds, Invent. Math. 145 (2001), no. 1, 151-218; MR1839289 (2002g:53158).

[16] J. Li, Stable morphisms to singular schemes and relative stable morphisms, J. Differential Geom. 57 (2001), no. 3, 509-578; MR1882667 (2003d:14066).

[17] D. McDuff and D. Salamon, Introduction to Symplectic Topology, Oxford Mathematical Monographs, Oxford Science Publications, 1995.

[18] T. Oda, Convex Bodies and Algebraic Geometry: An Introduction to the Theory of Toric Varieties, Ergbnisse der Mathematik und ihrer Grenzgebiete. 3. Folge, Bd. 15, Springer-Verlag, 1988.

[19] M. Reid, Chapters on Algebraic Surfaces, in Complex algebraic geometry (Park City, UT, 1993), 3-159, Amer. Math. Soc., Providence, RI, 1997; MR1442522 (98d:14049).

Département de Mathématiques et de Statistique,

Université DE MontrÉAL,

CP 6128 succ Centre-Ville, Montréal, QC H3C 3J7, Canada

E-mail address: shengda@dms.umontreal.ca

ReCeived February 18, 2005. 